\title{
Ars Longa. Kunst und Nachhaltigkeit
}

\author{
Verena Kuni
}

\section{Zusammenfassung}

Welchen Beitrag kann zeitgenössische Kunst zu einer sozial-ökologischen Nachhaltigkeitsperspektive leisten? Dieser Frage geht der Beitrag ausgehend von künstlerischen Projekten nach, die in Feldern operieren, in denen die drei Säulen des bekannten Nachhaltigkeits-Modells - Umwelt, Soziales und Wirtschaft - gleichermaßen relevant sind: in der Forstwirtschaft („Bäume pflanzen“) und in der Imkerei (,Bienen züchten“). So entstehen in der Kunst Bilder, die nicht nur zur Vermittlung entsprechender Einsichten in für ein nachhaltiges Denken und Handeln relevante Zusammenhänge beitragen, sondern darüber hinaus je auf ihre Weise zum aktiven sozial-ökologischen Engagement einladen.

Der nachstehende Beitrag basiert auf Überlegungen, die ursprünglich 2010 für den Einführungsvortrag zur Frankfurter Bürger-Universität „Vorsorgen für die Welt von morgen - Positionen zur Nachhaltigkeit" formuliert wurden. ${ }^{1}$ An der interdisziplinären Vortragsreihe waren auch zahlreiche Kolleg*innen beteiligt, die

\footnotetext{
${ }^{1}$ Konzipiert und organisiert von der Verfasserin zusammen mit der Wissenschaftshistorikerin Anne Hardy-Vennen, dem Biologen und Wissenschaftsjournalisten Stephan M. Hübner (seinerzeit beide Goethe-Universität, Stabsstelle Presse \& Öffentlichkeitsarbeit) sowie der Biologin Heike Zimmermann-Timm (seinerzeit Leiterin der GRADE Graduierten-Akademie der Goethe-Universität); vgl. für Programmatik und Programm Goethe-Universität 2010, 8-18 sowie für ausgewählte Beiträge Forschung Frankfurt 2010.

V. Kuni $(\varangle)$

Institut für Kunstpädagogik der Goethe-Universität Frankfurt am Main, Frankfurt am Main, Deutschland

E-Mail: verena@kuni.org; kuni@kunst.uni-frankfurt.de
} 
ebenfalls mit Beiträgen im vorliegenden Band vertreten sind. Beides ist natürlich kein Zufall. Denn während „Nachhaltigkeit“ zu jenen Begriffen gehört, die - sicherlich nicht ganz grundlos - zum Basisvokabular professioneller und insbesondere politischer Phrasenproduktion gezählt werden beziehungsweise durch entsprechenden Ge- und Missbrauch an Leumund eingebüßt haben, liegt zugleich auf der Hand: Wer sich seriös für Nachhaltigkeitsthemen engagiert, wer theoretisch und/oder praktisch, wissenschaftlich und/oder künstlerisch im Feld arbeitet, wird dies kaum von Konjunkturen abhängig machen. Vielmehr ist bereits der Frage nach Nachhaltigkeit eine Zeitperspektive eingeschrieben, die auf eine langfristige Beschäftigung mit der Sache und auf die Ausdauer aller Beteiligten setzt.

Das bedeutet selbstverständlich nicht, einmal eingenommene Standpunkte zu zementieren, indem man sie wiederholt. Wohl aber kann es darum gehen, Wiederholung, Wiederaufnahme und Weiterführung miteinander zu verknüpfen: Das als Fundament zu belassen, was nach wie vor gültig erscheint, und nach geeigneten Ansatzpunkten zu suchen, auf die sich aufbauen lässt - wobei die neuerliche Betrachtung derselben Gegenstände ebenso zu neuen Überlegungen Anlass bieten kann wie neu hinzugekommene Gegenstände bereits gefasste Gedanken untermauern mögen. Und schließlich: Wem wäre es jemals gelungen, zweimal im selben Fluss zu baden?

In diesem Sinne mögen jene Leser*innen, die Grundgedanken und Passagen des vorliegenden Textes aus der früheren Lektüre kennen ${ }^{2}$, prüfen, ob es geglückt ist, die Wiedervorlage im neuen Kontext - mit der sich zudem die Gelegenheit bot, das bereits Bestehende zu überarbeiten, zu ergänzen und mit einem Apparat $\mathrm{zu}$ versehen - in der Weiterführung der seinerzeit entwickelten Stränge und unter Berücksichtigung aktueller Entwicklungen fruchtbar zu machen.

\section{$1 \quad$ Ars longa}

Vita brevis, ars longa - kurz ist das Leben, lang währt die Kunst: Wenngleich der Arzt Hippokrates, dem man den Aphorismus zuschreibt, seinerzeit kaum an die Bildende Kunst gedacht haben dürfte ${ }^{3}$, galt Letztere über Jahrhunderte hinweg als vornehmste Schöpferin und Verwalterin die Zeiten überdauernder Werte.

\footnotetext{
${ }^{2}$ Vgl. die Verschriftlichung des Beitrags zur Bürger-Universität, Kuni 2010a.

${ }^{3}$ Die im sogenannten „Corpus Hippocraticum“ - einer antiken Sammlung medizinischer Texte unterschiedlicher Provenienz - enthaltenen Aphorismen beziehen sich auf die Heilkunst; so auch der „1. Aphorismus“, dem die zitierten Worte entstammen.
} 
Heute hingegen scheint sich die Kunst in weiten Teilen aus einer solchen Perspektive verabschiedet zu haben - nicht nur, weil vorzugsweise in Materialien, Medien und Formaten gearbeitet wird, die kaum konservierbar sind. ${ }^{4}$ Angesichts der umfassenden Aufgaben, denen sich eine Politik der Nachhaltigkeit zu stellen hat, werden der Kunst weder der Einfluss noch die Kompetenzen zugebilligt, wie sie etwa zur Lösung drängender ökologischer und wirtschaftlicher Probleme vonnöten wären. Bestenfalls erwartet man von ihr, wirkmächtige Bilder für Utopien und Dystopien zu schaffen, Schreckensszenarien einer Endzeit zu zeichnen oder mit positiven Gegenentwürfen einem Wunsch nach Ganzheitlichkeit Ausdruck zu verleihen.

Doch nicht von ungefähr mehren sich die Stimmen jener, die Nachhaltigkeit nicht nur als gesamtgesellschaftliche Herausforderung verstehen, sondern gerade in Kultur und Künsten wichtige Säulen für zukunftsfähiges Denken und Handeln sehen. ${ }^{5}$ Zudem begnügen sich zeitgenössische Künstler*innen längst nicht mehr mit Beiträgen zu einer ökologischen oder sozialen Ästhetik. ${ }^{6}$ Zusammen mit Wissenschaftler*innen unterschiedlicher Disziplinen arbeiten sie an Projekten, die kreative Impulse für nachhaltige Entwicklungen mit konkreten Perspektiven für die Praxis verbinden - und zwar einer Praxis, die in den Projekten nicht nur exemplarisch vorgeführt und ,zur Nachahmung empfohlen“ wird ${ }^{7}$, sondern mitunter auch direkt zur aktiven Beteiligung einlädt.

Damit gewinnt auch die Rede von der ,,ars longa“ neuen Sinn. Der hippokratische Aphorismus lässt sich nämlich so deuten, dass sich aus der Kürze des Lebens gerade für die Kunst - die sich auf ein historisch über lange Zeiträume

\footnotetext{
${ }^{4}$ Überlegungen dazu, inwiefern entsprechende materiale und mediale Praktiken und Strategien in einem Zusammenhang mit eben jenen Fragestellungen stehen, mit denen sich dieser Beitrag beschäftigt, möchte ich an anderer Stelle weiterführend nachgehen.

${ }^{5}$ Vgl. für eine auf Kunst als Impulsgeberin nachhaltiger Entwicklung ausgehende Sammlung von Positionen, die insbesondere interdisziplinäre, partizipative und ganzheitlichbildungsorientierte Ansätze ins Auge fasst, Kurt und Wagner 2002; für einen weiterführend methodisch-systematisch argumentierenden Ansatz Kagan 2011.

${ }^{6}$ Weder impliziert noch intendiert dieser Hinweis eine Abwertung entsprechender Beiträge, zumal beide Konzepte sowohl für sich genommen als auch im Kontext der im Folgenden betrachteten Zusammenhänge Wichtiges leisten; vgl. zur ökologischen (Natur-)Ästhetik grundlegend Böhme 1989; für eine Überblicksdarstellung klassischer Ansätze und Positionen Strelow 2004; weiterführend Miles 2014; zur sozialen Ästhetik grundlegend Olander 1983 (siehe Deitcher 2010); weiterführend Bradley und Esche 2007.

${ }^{7}$ In Anlehnung an das für den hier diskutierten Komplex in bestem Sinne exemplarische Ausstellungsprojekt gleichen Titels, das von Adrienne Goehler konzipiert und realisiert wurde; vgl. Goehler 2010 sowie https://www.z-n-e.info (Zugriff 20. Juni 2020).
} 
gesammeltes Wissen berufen kann und zugleich auch stets über den Moment hinaus auf eine Zukunft hin denken muss - eine besondere Verantwortung ergibt, im Hier und Jetzt tätig zu werden. Im Übrigen wird im - meist nicht mit zitierten weiteren Verlauf des Aphorismus auch darauf verwiesen, dass keineswegs allein der Arzt in der Verantwortung steht: „der Kranke selbst und seine Umgebung, eben so wie die äussern Umstände müssen, jeder das Seinige, zur Erreichung des Zweckes beitragen." (Boenninghausen 1863, S. 1).

Ausgehend von Joseph Beuys - einem der ersten und wohl auch prominentesten deutschen Künstler, der sich in seiner Arbeit explizit auf das Ineinanderwirken ökologischen, sozialen und wirtschaftlichen Denkens und Handels bezogen hat, wie es für den hier zugrunde gelegten Nachhaltigkeitsbegriff eine zentrale Rolle spielt $^{8}$ - sollen im Folgenden exemplarische Projekte vorgestellt werden, die einen Einblick in das Spektrum der Ansätze bieten, die auf eine solche Kunst der Nachhaltigkeit abzielen.

Mit Blick auf die in diesem Zusammenhang relevanten lokalen, regionalen und globalen Dimensionen hatten bereits in der ersten Fassung der vorliegenden Überlegungen Künstler*innen im Mittelpunkt der Betrachtung gestanden, deren Arbeiten genau diese Dimensionen nicht nur für sich genommen ausloten, sondern insbesondere auch aus der Perspektive und für den Diskussionskontext anschaulich zu machen geeignet schienen, in denen sich Vortragende und Teilnehmer*innen 2010 und $2017^{9}$ bewegten. Die Stadt Frankfurt, das RheinMain-Gebiet, Hessen, Deutschland, Europa, die sogenannte westliche Welt und die sogenannte nördliche Hemisphäre, die Welt: Schon die Aufzählung mag darauf verweisen, dass es sich zwar immer auch, aber nie allein um geografische Koordinaten handelt, in denen wir uns positionieren und in denen wir navigieren - sondern vielmehr um auf vielfältige und komplexe Weise kulturell, sozial, historisch, politisch, ökonomisch und ökologisch konditionierte Lebensräume.

Zugleich zeigt sie an, dass es stets konkrete Ausgangspunkte, Anlässe, Kontexte und Radien des Wahrnehmens, Denkens, Handelns gibt, „die eigene

\footnotetext{
${ }^{8}$ Seit Anfang/Mitte der1990er Jahren prominent im ,Drei-Säulen-Modell der nachhaltigen Entwicklung“ geführt, letztlich aber auch schon sehr viel früher bestimmend, scheint die grundlegende Verflochtenheit der drei Komplexe und ihre Relevanz für ,welchen Nachhaltigkeitsbegriff auch immer" unbestreitbar - während dessen Füllung stark von der Interpretation des Modells und der Frage abhängt, von welchen Interessen geleitet und in welche Richtungen dieses Beziehungsgeflecht, seine Entwicklung und seine Transformationen handlungsorientiert gedeutet werden. Vgl. weiterführend zur historischen Perspektive Grober 2010.

${ }^{9}$ Angesprochen sind hier die Frankfurter Bürger-Universität im Sommersemester 2010 sowie die Vortragsreihe von GRADE Sustain 2016-2017 bzw. meine Vorträge im Rahmen dieser beiden Veranstaltungen.
} 
Haustüre, vor der es zu kehren gilt", die individuellen Kompetenzen, die sich einbringen lassen, die eigene Perspektive, die auf spezifische Weise geprägt ist - dass diese aber stets in einem größeren Zusammenhang von Voraussetzungen, Konditionen, Interaktionen und Konsequenzen stehen.

Letzteres wiederum mag bereits einen Hinweis darauf geben, warum es Künstler*innen - denen man zunächst einmal besonders dann, wenn es um Aufgaben und Probleme globalen Ausmaßes wie jene, die auch in den aktuellen Debatten um Nachhaltigkeit eine zentrale Rolle spielen, eher geringeren Einfluss und weit weniger Handlungsmacht zubilligen wird als Akteur*innen aus Wirtschaft und Politik - wagen, mit ihren Arbeiten Stellung zu beziehen und sich mit ihren Projekten im Feld engagieren. Wie im Folgenden noch näher auszuführen sein wird, hat dies durchaus auch mit den spezifischen Kompetenzen und Potenzialen der Kunst zu tun: nämlich Bilder zu schaffen, die - wortwörtlich nachhaltig - zum Denken und Handeln anregen.

Dies festzustellen heißt weder, der für die Moderne durchaus prägsamen „Kunstreligion“ ein auf das ausgehende Anthropozän zugeschnittenes Kapitel hinzufügen zu wollen, indem man die Aufgabe, Alternativen zum Bestehenden aufzuzeigen, vorzüglich den Künsten zuweist und sie zum Hoffnungsträger für Heilserwartungen stilisiert. Noch auch soll behauptet werden, dass jegliche Art von Kunst mit einschlägigen Bezügen das Potenzial besitzt und/oder intentional darauf ausgerichtet wäre, gesellschaftliche, ökologische und/oder ökonomische Missstände nicht nur zu kritisieren, sondern auch konkrete, konstruktive Impulse zur Korrektur dieser Missstände zu vermitteln. ${ }^{10}$

In jedem Fall jedoch lässt sich Adrienne Goehlers Hinweis folgen, dass es nicht nur angemessen ist, den drei Nachhaltigkeitsdimensionen Gesellschaft, Wirtschaft und Umwelt mit der Kultur eine vierte hinzuzufügen - sondern auch lohnend, sich eingehender mit den Impulsen $\mathrm{zu}$ befassen, die diese für nachhaltiges Denken und Handeln bereit hält (vgl. Gersmann und Wilms 2010, S. 8).

\section{Eichen}

Einen Baum pflanzen: Nicht von ungefähr zählt dies zu den Handlungen, die schon der Volksmund mit Nachhaltigkeit verknüpft. Wer einen Baum pflanzt, ein

\footnotetext{
${ }^{10}$ Siehe hierzu auch die kritischen Überlegungen von Sacha Kagan zu dem, was er - in einer Schärfe, der selbst aus der Perspektive einer kritischen Kunstwissenschaft sicher nicht so durchgängig zu folgen ist - als „The Culture and Art of Unsustainability“ bezeichnet; vgl. Kagan 2011, 23-92.
} 
Haus baut, ein Kind zeugt, will ,,vorsorgen für die Welt von morgen“"11, auch über die eigene Lebenszeit hinaus.

Als der Künstler Joseph Beuys 1982 im Rahmen der siebten documenta jener Großausstellung, die alle fünf Jahre aus dem nordhessischen Kassel eine „Weltstadt der Kunst“ macht ${ }^{12}$ - zum Spaten griff, um direkt vor dem Museum Fridericianum eine Eiche zu pflanzen, ging es ihm um ebendies. Unter dem Motto „Stadtverwaldung statt Stattverwaltung“ trat er in seiner Aktion „7000 Eichen“ an, mit den Mitteln der Kunst für ein nachhaltiges Denken und Handeln aller zu werben. ${ }^{13}$ Und er wusste seinen Wirkungskreis zu nutzen, um diesem Anspruch Nachdruck zu verleihen. Nicht nur begleitete er sein Projekt mit Vorträgen, Diskussionen und weiteren, bildmächtigen Aktionen - darunter der "Schmelzaktion“, in deren Zuge Beuys zwei Wochen nach der Eröffnung der Schau auf dem Friedrichsplatz einen Ofen errichtete, um als „Künstler-Alchemist“ die wertvolle Nachbildung einer Zarenkrone in das Ensemble „Friedenshase“ und „Sonnenkugel“" zu transformieren. ${ }^{14}$ Vor allem hatte er es von Anfang an so angelegt, dass die Stadt Kassel und ihre Bürger*innen in Zugzwang waren: Für seinen documenta-Beitrag hatte er sich ausbedungen, einen Keil aus 7000 Basaltstelen auf dem zentralen Friedrichsplatz aufzuschütten, der nunmehr Stück um Stück abzutragen war: Mit einer Spende von 500 DM erwarb man das Recht, selbst eine Eiche zu pflanzen, der dann eine der Basaltstelen beigesellt wurde. Nicht allein wegen der erheblichen Kosten für die Umsetzung ${ }^{15}$, sondern auch schon wegen der Standortsuche für die Bäume erwies sich das Projekt als langwieriger Prozess. Die zunächst letzte der mit den von Beuys vor Ort ausgebrachten Basaltstelen zu

\footnotetext{
${ }^{11}$ In Anlehnung an den Titel der Vortragsreihe zur Frankfurter Bürger-Universität 2010; vgl. Goethe-Universität Frankfurt 2010.

${ }^{12}$ Zwar wird diese - sonst auf Paris, London, New York, Florenz oder Berlin gemünzte - Wendung vorzugsweise vom Stadtmarketing genutzt. Der Gedanke, dass Kunst (und kulturelle Bildung) Menschen zu mindestens ideellem Kosmopolitanismus und zu Weltverständnis verhelfen, spielte jedoch schon für den documenta-Gründer Arnold Bode eine zentrale Rolle. Zur Geschichte der documenta vgl. Kimpel 1997; Schwarze 2012 sowie Eichel 2015.

${ }^{13}$ Vgl. zum Projekt: Groener und Kandler 1987; Stiftung 7000 Eichen 2012; die quellenreiche Dokumentation in Loers und Witzmann 1993, 221-283 sowie https://www.7000eichen.de Zugriff 20. Juni 2020.

${ }^{14}$ Zur Aktion und ihrer Deutung vgl. ausführlich Kuni 2006, Bd. I, insb. Kap. III.9., 503-509.

${ }^{15}$ Die Kosten für die erste Realisierungsphase werden auf 4,3 Mio. DM geschätzt, was das Produktionsbudget der documenta um ein Vielfaches überstieg; für die Erbringung unternahmen zunächst Beuys selbst und in der Folge der 2002 in eine Stiftung überführte Verein „7000 Eichen“ erhebliche Anstrengungen, Projektgelder und Spenden einzuwerben.
} 
paarenden „7000 Eichen“"16 wurden 1987 - ein Jahr nach dem Tod des Künstlers - zur documenta 8 von seinem Sohn neben die erste Eiche gepflanzt.

Indes schieden und scheiden sich an dem Projekt auch weiterhin die Geister. So manche*r Kunstliebhaber*in konnte der Aktion wie auch insgesamt dem politischen Engagement des Künstlers, der 1979 sogar als Direktkandidat der Grünen für das Europa-Parlament kandidiert hatte (vgl. Beuys 1978) ${ }^{17}$, kaum etwas abgewinnen. Die Kasseler*innen haben selbst nach dem Abtragen des Basaltkeils nach wie vor ihre Mühen und Kosten mit dem Projekt, nicht nur, weil die Baumpflanzungen verschiedentlich Vandalismus zum Opfer fallen - wobei insbesondere die Basaltstelen aus ästhetischen wie sicherheitstechnischen Gründen ,nachhaltig * Anstoß erregen. Wie jede Stadt befindet sich auch Kassel kontinuierlich in Veränderung; Bäume müssen Bauvorhaben weichen oder erkranken - ein Problem, mit dem gerade auch Stadtbäume durch die ökologische Mehrfachbelastung, zu der lokale Luftverschmutzung ebenso wie Klimafaktoren zählen, in zunehmendem Maße konfrontiert sind. ${ }^{18}$

Wenngleich die Idee der "Stadtverwaldung“ direkt an die historischen Wurzeln des Nachhaltigkeitsgedankens und dessen Begriffsgeschichte im deutschen Sprachraum anzuknüpfen scheint, die in der Forstwirtschaft liegen ${ }^{19}$, mag man

16 Tatsächlich wurden nicht ausschließlich Eichen gepflanzt, sondern neben Stiel-, Sumpfund Roteichen mehr als dreißig weitere Baumarten, darunter etwa auch Gingko biloba. Die Standorte der Bäume sind in einem Baumkataster verzeichnet und über das Geoportal der Stadt Kassel einsehbar; vgl. https://geoportal.kassel.de/portal/apps/webappviewer/index.html?id= ada987a713c54778bd5beecbc89861c9 bzw. eingebunden in die Projektseite „7000 Eichen“ https://www.7000eichen.de/index.php?id=20 Zugriff jeweils 20. Juni 2020.

17 Anlässlich der Kandidatur 1979 wurde der Text separat wieder aufgelegt und breit multipliziert; vgl. via Wilfried Heidt https://www.wilfried-heidt.de/beuys-heidt-zusammenarbeit/ pdf/Aufruf-zur-Alternative-Heft.pdf Zugriff 20. Juni 2020.

${ }^{18}$ So laufen seit einigen Jahren auch in mehreren Bundesländern Forschungsprojekte, die sich mit den veränderten Standortbedingungen speziell für Stadtbäume sowie damit befassen, welche Baumarten unter den Konditionen des Klimawandels bessere Überlebenschancen haben als jene, die bislang gepflanzt wurden; vgl. z. B. das bayerische Projekt, ,Stadtgrün 2021: Neue Bäume braucht das Land!“, https://www.lwg.bayern.de/landespflege/urbanes_gruen/085113/ index.php Zugriff 20. Juni 2020; die Forschungen am Frankfurter Senckenberg Forschungszentrum für Biodiversität und Klima SBiK-F https://www.bik-f.de Zugriff 20. Juni 2020; die exemplarische Untersuchung von Gillner 2012; zur Rolle der Klimafaktoren Mosbrugger et al. 2012; sowie zu weiteren Forschungs- und Kunst-Projekten im Feld weiterführend auch unten.

${ }^{19}$ Erstmals begegnet der Begriff in einschlägigem Kontext in Hans Carl von Carlowitz „Sylvicultura oeconomica, oder haußwirthliche Nachricht und Naturmäßige Anweisung zur wilden Baum-Zucht“ (Carlowitz 1713); hier allerdings steht der wirtschaftliche Zweck der Ressourcenschonung im Vordergrund. Vgl. weiterführend Grober 2010, 111-121. 
sich schließlich fragen, warum Beuys ausgerechnet Eichen in den Stadtraum pflanzen wollte und warum er seine Aktion im vergleichsweise grünen Kassel beziehungsweise im (eichen-)waldreichen Nordhessen situierte.

Die Antwort ist einfach: Als Künstler dachte Beuys in Bildern. Vor diesem Hintergrund hatte er sich bewusst für die Eiche als einen historisch konnotierten Baum von monumentalem Wuchs und sprichwörtlich langer Lebensdauer entschieden, dem er das in die Erde eingesenkte, erstarrte Vulkangestein als Konterpart zur Seite stellte. ${ }^{20}$ Mindestens ebenso wichtig wie die Dimension der Zeit und der ökologische Aspekt des Stadtgrüns war ihm jedoch das, was er als „Soziale Plastik“21 bezeichnete: Die Pflanzung eines Baums in der und für die Gemeinschaft als exemplarischer Akt sozialen Handelns, zu dem auch die Übernahme von Verantwortung und das Aushandeln von Konflikten gehören. Die Kasseler documenta bot ihm als international beachtete Ausstellung eine denkbar geeignete Plattform für sein Projekt.

Im Übrigen scheint die Zeit Beuys in mehrfacher Hinsicht Recht zu geben. In Kassel hat sich der Unmut der Skeptiker*innen und Gegner*innen weitgehend gelegt. Unter jenen, die sich um den Erhalt und die Pflege der Bäume kümmern, finden sich heute neben der Stadt und der eigens gegründeten Stiftung „7000 Eichen“ nicht nur kulturell und ökologisch engagierte Bürger*innen, sondern auch ortsansässige Firmen, die im Feld der nachhaltigen Technologien tätig sind (vgl. Stiftung 7000 Eichen 2012). In der Folge wurden zudem in weiteren Städten „Beuys-Eichen“ und andere Bäume unter den Vorzeichen der Kunst gepflanzt. ${ }^{22}$

\section{Bäume pflanzen $\mathbf{2 . 0}$}

Eine unmittelbare Hommage an Beuys' Projekt ist unterdessen im Internet entstanden: 2007 hat das italienische Künstler*innen-Duo Eva und Franco Mattes,

${ }^{20}$ Vgl. zur Bedeutung der Basaltstelen, die Beuys auch in weiteren Werken dieser Zeit verwendete, insbesondere in Kombination mit dem (Eich-)Baum weiterführend Kuni 2006, Bd. I, Kap. III.9., 510-514.

${ }^{21}$ Vgl. zum Begriff und seinen Dimensionen im Werkkontext Harlan, Rappmann und Schata (1976) 1980.

${ }^{22}$ So unter anderem im Rahmen von „7000 Oaks“ am DIA Center New York, begonnen 1988 und ab 1995 fortgesetzt; vgl. https://www.diaart.org/visit/visit/joseph-beuys-7000-oaks, Zugriff 20. Juni 2020, und mit dem seit 1989/1990 laufenden Projekt „BAUMKREUZ“, das von Beuys ‘ ehemaligem Studenten Johannes Stüttgen, dem Landschaftsarchitekten Norbert Scholz, dem Kurator Konstantin Adamopulos und dem Unternehmer Frank Wilhelmi initiiert wurde; vgl. https://www.bund-thueringen.de/gruenes-band/baumkreuz/Zugriff 20. Juni 2020. 
das in den 1990er Jahren mit Netzkunst Aufsehen erregte und sich seither konsequent auf künstlerische Interventionen spezialisiert hat, die sich mit den Entwicklungen in der digitalen Technologie und Medienkultur befassen ${ }^{23}$ (vgl. Quaranta 2009), eine digitale Version von „7000 Eichen“ für die 3D-onlinePlattform „Second Life“ erstellt. In einer ganzen Werkreihe befassten sich die beiden seinerzeit mit von ihnen alternativ auch als „Synthetic Performances“ bezeichneten „Reenactments“ - Software-basierten „Wiederaufführungen“ beziehungsweise Adaptionen prominenter Peformances aus der zweiten Hälfte des 20. Jahrhunderts in digitalen online-Umgebungen. ${ }^{24}$ Für ,Joseph Beuys' 7000 Oaks“" waren zunächst mit der für die Fertigung digitaler Objekte vorgesehenen „Second Life“-Software 7000 ,Eichen“ und 7000 „Basaltsteine“ entstanden; am 17. März 2007 - genau fünfundzwanzig Jahre nach Beuys“ initialer Baumpflanzung in Kassel - wurden von den Avataren der beiden Künstler*innen die ersten Eiche-Stein-Paare in „Second Life“ gesetzt, während Nutzer*innen der Plattform eingeladen waren, sich in der Folge selbst an der virtuellen Pflanzaktion zu beteiligen. ${ }^{25}$ Im Sommer 2008 wurde das Projekt noch einmal prominent positioniert, indem es als erster künstlerischer Beitrag für die neue Präsenz des GoetheInstituts in „Second Life“ figurierte, zu der auch Künstler*innen-Residenzen für die Entwicklung eigens auf die Medienplattform zugeschnittener Arbeiten gehören sollten. ${ }^{26}$

Nun kann ein solches Reenactment sicher dazu beitragen, einer jüngeren, medienaffinen Generation die Grundgedanken von Beuys' Baumpflanzungsaktion zu vermitteln und sie für das ursprüngliche Projekt zu interessieren. Für sich

${ }^{23}$ Vgl. für einen Werküberblick die Webseite des Künstler*innen-Duos, https://010010111 0101101.org, Zugriff 20. Juni 2020.

${ }^{24}$ Zur Reihe der „Reenactments“ (2007-2010), die als Live-Events im Rahmen von Ausstellungen und zugleich im Netz stattfanden, gehör(t)en bekannte Arbeiten von Gilbert \& George, Vito Acconci, Chris Burden und VALIE EXPORT; vgl. https://0100101110101101.org/ree nactments/ Zugriff 20. Juni 2020.

${ }^{25}$ Anders als die anderen „Reenactments“ ist „Joseph Beuys“ 7000 Oaks“ nicht mehr auf der Webseite des Künstler*innen-Duos aufgeführt; die ursprüngliche URL der Dokumentation, https://www.0100101110101101.org/home/reenactments/performance-beuys.html, generiert eine Fehlermeldung, Zugriff 20. Juni 2020.

${ }^{26}$ Zur „Second Life“-,Insel“ des Goethe-Instituts, die von 2008 bis 2014 existierte, gehörten u. a. eine Bühne, Ausstellungsräume und ein Café; vgl. für eine Dokumentation https://www.bokowsky.net/de/referenzen/goethe_institut/second_life/insel/index.php Zugriff 20. Juni 2020. Da sich gerade in den ersten Jahren nach dem offiziellen Release von „Second Life“ 2003 zahlreiche Künstler*innen mit den Potenzialen der Plattform befassten, war es durchaus konsequent für eine mit kultureller Bildung befasste Institution, entsprechende Projekte einzuladen. 
genommen muss eine solche mediale Emulation auch keineswegs gegen Konzepte ausgespielt werden, die demgegenüber auf das Pflanzen ,echter' beziehungsweise biologischer Bäume in einer im weitesten Sinne ,,natürlichen Umwelt“27 setzen: Im Gegenteil mögen gestalterische Aktivitäten in digitalen Räume nicht nur generell zur Entwicklung und Wertschätzung kreativer Fähigkeiten und Tätigkeiten beitragen, sondern in diesem Fall auch spezieller zur Reflexion von Fragen der Landschafts- und Umweltgestaltung anregen ${ }^{28}$ - und spätestens dann, wenn die digitalen Gärtner*innen feststellen, dass das künftige Schicksal ihrer Pflanzungen gegebenenfalls davon abhängen kann, ob die Plattform und deren Zugänge, die Werkzeuge und die Codes offen bzw. quelloffen oder proprietär sind, dürfte es hinreichend Motivationen zu Vergleichen und Erfahrungen mit Praktiken im analogen Raum geben. ${ }^{29}$

Mit Blick auf die Energiebilanz sind jedoch gerade in Sachen Nachhaltigkeit bei computer- und netzbasierten Projekten deutliche Abstriche zu machen: Tatsächlich trägt unsere Nutzung digitaler Technologien ganz erheblich zur Vergrößerung des ,„ökologischen Fußabdrucks“ bei. ${ }^{30}$ Zudem gilt es nicht zu vergessen: über das Internet lassen sich zwar Informationen weltweit verbreiten - nachhaltiges Handeln, auch im Umgang mit und in der Nutzung von Hardund Software, findet primär im Realraum statt; hier wiederum sind, gerade im Bewusstsein um die letztlich immer globalen, grenz- und systemübergreifenden Zusammenhänge und Konsequenzen einzelner Handlungen und ihrer Effekte, individuelle und kollektive lokale Initiativen von entscheidender Bedeutung.

\footnotetext{
${ }^{27}$ Während dieser Begriff prinzipiell durchaus auch auf Baumpflanzungen in insgesamt durchgestalteten urbanen Räumen, aber auch auf komplett von Menschenhand angelegte Forste Anwendung finden kann, mögen diese zugleich darauf verweisen, wie schwierig es mit Blick auf Mensch-Umweltbeziehungen ist, „Natur“" und „Natürlichkeit“ von „Artifizialität“ bzw. „Künstlichkeit“" abzugrenzen.

${ }^{28}$ Wenngleich sich künstlerische Projekte wie das von Eva und Franco Mattes eher selten als Beiträge zum „E-Learning“ oder „Serious Gaming“ verstehen und ihnen dementsprechend nicht notwendigerweise explizit hierauf ausgerichtete didaktische Konzepte zu Grunde liegen, scheint es doch nicht ganz falsch, in den von ihnen gesetzten Impulsen entsprechende Potenziale auszumachen.

${ }^{29}$ Letztere sind freilich, wie nicht nur das Schicksal mancher „Beuys-Eiche“ belegt, mitunter nicht weniger dem Zugriff Dritter ausgesetzt.

${ }^{30}$ Vgl. einführend zur Energieproblematik De Decker 2009; zum Abfall Ogunseitan u. a. 2009; weiterführend Cubitt 2017 sowie das künstlerisch-wissenschaftliche Forschungsprojekt „Times of Waste“ (FHNW Hochschule für Gestaltung und Kunst Basel, 2015-2018), aus dem u. a. auch eine Wanderausstellung (2018-2020 f.) hervorgegangen ist, s. https://times-of-was te.ch Zugriff 20. Juni 2020.
} 
Das musste auch Dirk Fleischmann feststellen, als er 2007 vom Karlsruher Zentrum für Kunst und Medien $(\mathrm{ZKM})^{31}$ und dem von der Royal Society for the encouragement of Arts, Manufactures and Commerce (RSA) gemeinsam mit dem Arts Council England initiierten RSA Arts and Ecology Centre London ${ }^{32}$ eingeladen wurde, ein Projekt mit Ökologie-Bezug für die ZKM-Repräsentanz in „Second Life“" zu entwickeln.

Schon zu seiner Studienzeit an der Frankfurter Städelschule hatte der Künstler damit begonnen, sich mit Nachhaltigkeitsfragen zu beschäftigen. So betrieb er in der Akademie einen Kiosk mit Süßigkeiten, für die seine Kommiliton*innen entweder den regulären Preis bezahlen oder einen Obolus nach Gusto entrichten konnten. ${ }^{33}$ Den Gewinn reinvestierte Fleischmann in neue Ware; die VerpackungsDisplays sammelte er, sortierte und stapelte sie in seinem Atelier. Am Ende des Semesters konnte er beim Rundgang jeweils eine beeindruckende Rauminstallation präsentieren. Aus der Untersuchung studentischer Ökonomie wurde so nebenbei Reycling-Kunst, die denkbar anschaulich demonstrierte, wie viel Abfall allein der kleine Hunger zwischendurch produziert, wenn man nicht zu einem Butterbrot oder einem Stück Obst, sondern zu Schokoriegeln und anderen Fertigsnacks greift. Den monetären Erlös aus seinem Kiosk und aus weiteren Projekten wie „mychickeneggproduction“ $(2001)^{34}$ - einem Gehege für freilaufende Hühner, dessen Gestaltung auf bis dahin lediglich als Konzept existierenden Plänen der Kölner Künstlerin Rosemarie Trockel basierte - setzte Fleischmann ein, um 2004 auf dem Dach der Städelschule Solarpanels zu installieren. ${ }^{35}$

Für sein „Second Life“-Projekt hatte sich der Künstler den $\mathrm{CO}_{2}$ Emissionshandel als Thema gewählt und geplant, eine Baumpflanzung vorzunehmen, die den ,ökologischen Fußabdruck“ der virtuellen ZKM-Repräsentanz zugleich sichtbar machen und kompensieren sollte. Für jeden realen Baum wollte er wiederum einen digitalen Baum pflanzen und mit Informationen über die $\mathrm{CO}_{2}$-Emissionen verknüpfen. Schon bald sah er sich jedoch mit zahlreichen Problemen konfrontiert: Als Ort für die Pflanzungen hatte er die Philippinen

\footnotetext{
${ }^{31} \mathrm{Vgl}$. zur Institution https://zkm.de/ Zugriff 20. Juni 2020.

${ }^{32}$ Das RSA Arts and Ecology Centre bestand von 2005 bis 2010 ,,as a catalyst for the insights, imagination and inspirations of artists in response to the unprecedented environmental challenges of our time, with a focus on their human impact", vgl. https://www.thersa.org/actionand-research/rsa-projects/design/arts-and-ecology Zugriff 20. Juni 2020.

${ }^{33}$ Vgl. das Projekt ,mykiosk“ (1998-2002), dokumentiert auf der Webseite des Künstlers: https://dirkfleischmann.net/mykiosk Zugriff 20. Juni 2020.

${ }^{34} \mathrm{Vgl}$. https://dirkfleischmann.net/mychickeneggproduction Zugriff 20. Juni 2020.

${ }^{35} \mathrm{Vgl}$. https://dirkfleischmann.net/mysolarpowerplant Zugriff 20. Juni 2020.
} 
ausgewählt, die als Gegenleistung für Aufforstungsmaßnahmen Emissionszertifikate anbieten. Aber allein über das Internet ließ sich weder die Pflanzung noch die Beauftragung einer dortigen Firma mit der Programmierung der ,Second Life"-Bäume organisieren. Zudem wäre die Pflanzung weniger einzelner Bäume lediglich eine symbolische Geste geblieben. Daher ließ sich Fleischmann auf das Wagnis eines weitaus umfangreicher angelegten Aufforstungsprojekts ein. Er reiste selbst auf die Philippinen und gewann dortige Umwelt-Engagierte und Bäuer*innen für die Realisierung. So entstand eine echte „Forest Farm“, über deren Fortschritte die lokale Betreibergemeinschaft in den ersten Jahren der Laufzeit des Projekts regelmäßig im World Wide Web berichtete ${ }^{36}$ - indes in ,Second Life" ein schlichtes Bauschild genügte, das über das Schicksal des Projekts informierte. ${ }^{37}$

Ungeachtet der vergleichsweise sparsamen Nutzung von Netzressourcen sind Dokumentation und künstlerischer Output des Projekts allerdings nicht durchgängig konsequent auf einen nachhaltigen Umgang mit Technologie ausgerichtet. So entstand 2010 die Reihe ,mycarboncredits“, die auf 1838 digitalen Fotografien von seinerzeit 1838 Bäumen der ,myforestfarm“ basiert. Für jede dieser BaumFotografien wurde eine eigene Foto-CD gebrannt, deren schimmernde Oberfläche Dirk Fleischmann fotografierte, sodass am Ende eine Reihe von 1838 scheinbar ungegenständlichen Farbaufnahmen stand. ${ }^{38}$ Diese wurden als jpg-Dateien archiviert und werden im Ausstellungskontext auf kleinen Bildschirmen präsentiert; ebenfalls 2010 zeigte sie Fleischmann in Form einer großformatigen Videoprojektion mit dem Titel „A Walk In A Forest“ im Rahmen des Gwangju Media Arts Festival im öffentlichen Raum. ${ }^{39}$ Zweifelsohne können beide Arbeiten beziehungsweise Präsentationsformate ästhetisch und konzeptuell überzeugen: Der mehrfache Transfer zwischen den Dimensionen, in denen Baum und Raum zusammenkommen und sich manifestieren, schließen unmittelbar an die Eckpunkte des ursprünglichen Projektes an - und wenn man will, mag man sogar eine visuelle Analogie zwischen den Baumringen und dem ebenfalls konzentrisch aufgebauten physikalischen Speichermedium erkennen. Zugleich besitzt Letzteres eine geringere Lebensdauer als ein Baum - und trägt ganz im Gegensatz zu diesem von der Herstellung bis zur Entsorgung als Technoschrott zur ökologischen

\footnotetext{
${ }^{36}$ Vgl. https://www.myforestfarm.com/ Zugriff 20. Juni 2020.

${ }^{37}$ Vgl. für einen Screenshot der Tafel in ,Second Life“ die Dokumentation auf der alten, inzwischen archivierten myforestfarm-Webseite https://2008.myforestfarm.com/art.html Zugriff 20. Juni 2020.

$38 \mathrm{Vgl}$. https://dirkfleischmann.net/mycarboncredits Zugriff 20. Juni 2020.

${ }^{39}$ Vgl. https://dirkfleischmann.net/gwangju-media-arts-festival Zugriff 20. Juni 2020.
} 
Belastung bei. Verglichen mit dem nachhaltigen Erfolg des Kernprojekts fällt eine solche Teilbilanz freilich kaum kritisch ins Gewicht.

Insgesamt lässt sich Dirk Fleischmanns „,myforestfarm“ in ihrer Verschränkung von künstlerischem Konzept und ökologischem, ökonomischem sowie sozialem Handeln zweifelsohne als eine zeitgemäße Nachfolge von Beuys' „7000 Eichen“ sehen. Zugleich belegt das Projekt in ganz ähnlicher Weise wie die Kasseler „Stadtverwaldung“, aber etwa auch das 2001 vom Fotografen Sebastião Salgado auf dem Grundbesitz seiner Familie begonnene Wiederaufforstungsprojekt „Bulcão Farm“40 zweierlei: Aus der Initiative und dem Engagement einzelner Menschen und kleiner Gemeinschaften kann Großes entstehen - und die Kunst ist dabei nicht nur eine guter Kommunikatorin, sondern dürfte schon vorweg den Weg zum Ziel auf entscheidende Weise mit gebahnt haben: Künstler*innen sind Profis darin, mit wenigen Mitteln viel zu erreichen - und jene Mittel, die sie benötigen, einzuwerben. Und sie lernen früh, die Freiräume, die dem scheinbar so marginalen künstlerischen und kulturellen Handeln offen stehen, gerade weil man ihm in der Regel weniger Relevanz zuschreibt als etwa ökonomischem und wirtschaftlichem Handeln, höchst effizient zu nutzen.

Derweil gibt es in Deutschland auch wieder forstwirtschaftliche Projekte, in denen die Eiche eine zentrale Rolle spielt: Etwa den ${ } \mathrm{CO}_{2}$-Speicher Eichenwald“, den die Technische Universität München 2008/2009 zusammen mit dem Bayerischen Staatsforstamt angelegt hat. Gefördert wurde die Pflanzaktion vom Autohersteller Audi, der die Bäume jeweils in der Nähe verschiedener Produktionsstandorte setzen ließ. In diesem Zuge wurden nahe Ingolstadt in einem ehemals von Nadelhölzern dominierten Areal, dessen Bestände durch Windbruch und Borkenkäferbefall vollständig zerstört worden waren, 36.000 Stieleichen gepflanzt, die den Klimaveränderungen trotzen sollen. ${ }^{41}$ In Hessen wiederum haben im Rahmen des am Frankfurter Senckenberg Biodiversität und KlimaForschungszentrum (BiK-F) sowie der Goethe-Universität angesiedelten Projekts

\footnotetext{
${ }^{40}$ Vgl. für das von Salgado gemeinsam mit seiner Lebensgefährtin Lélia Deluiz Wanick begründete und inzwischen in die Stiftung „Instituto Terra“ überführte Projekt dessen Webseite https://www.institutoterra.org/ Zugriff 20. Juni 2020; Bekanntheit hat es hierzulande nicht zuletzt durch Wim Wenders“ Dokumentarfilm über Salgado erlangt, ,The Salt of the Earth“ (Regie: Wim Wenders, Juliano Ribeiro Salgado; F/BR 2014); vgl. https://www.dassal zdererde-derfilm.de/ Zugriff 20. Juni 2020.

${ }^{41}$ Die ab 2008 vorgenommenen Pflanzungen dienten als eine der Grundlagen des am TUMLehrstuhl für Waldwachstumskunde angesiedelten ,NELDER-Projekt Eiche: Biodiversität Produktivität und Kohlenstoff-Bindung von Eichenbeständen“ (2011-2019), das wiederum von der Audi Umweltstiftung gefördert wird; vgl. https://www.waldwachstum.wzw.tum.de/ forschung/projekte/nelder-eiche-audi/ Zugriff 20. Juni 2020.
} 
„Wald der Zukunft“ aus wärmeren Regionen stammende Eichenarten auf hessischem Boden eine neue Heimat gefunden; fortgesetzt wird die Forschung seit einiger Zeit im „South Hesse Oak Project (SHOP/FUTUREOAKS)““. ${ }^{42}$

Künstler*innen und andere Kulturschaffende mischen sich auch weiterhin aktiv und kreativ in dieses Forschungsfeld ein: Beispielsweise in Anlehnung an und/oder Auseinandersetzung mit im Wald situtiere(n) UmweltmonitoringProjekte(n) wie „Treewatch“43, die mit Hilfe neuer Technologien darauf abzielen, eine Kommunikationsschnittstelle zwischen Bäumen und Menschen zu schaffen, über die der Wald seinen Notstand gleichsam selbst melden und auf diese Weise umso nachdrücklicher zu nachhaltigem Umwelthandeln auffordern kann. ${ }^{44}$ So etwa der Schweizer Künstler Markus Maeder, der seit 2011/2012 teils gemeinsam mit Forst- und Umweltwissenschaftler*innen - zur Sonifikation klimabedingter Umweltveränderungen an Bäumen forscht. ${ }^{45}$

Oder sie arbeiten mit Projekten, die mit nachhaltigen Zukunftsutopien unmittelbar an Beuys“ Aufruf zur „Stadtverwaldung statt Stadtverwaltung“ erinnern mögen - wie etwa die exemplarische „Verwaldung“ eines Fußball-Stadions in Klagenfurt ${ }^{46}$ oder der Vorschlag, auf dem Dach des Flughafens Berlin Tempelhof nach dessen Stilllegung einen Wald zu pflanzen. ${ }^{47}$ Beide Ansätze lassen sich

\footnotetext{
${ }^{42}$ Vgl. die Basisinformationen auf den Webseiten des Institutes für Institut für Ökologie, Evolution und Diversität, Abt. Brüggemann https://www.bio.uni-frankfurt.de/47841321/For schung sowie weiterführend beim BiK-F https://www.bik-f.de/root/index.php?page_id=40 Zugriff jeweils 20. Juni 2020.

${ }^{43}$ Vgl. https://treewatch.net/ Zugriff 20. Juni 2020. Mit einem vergleichbaren Ansatz arbeitet auch das aus dem NELDER-Projekt hervorgegangene Münchener Eichen-Projekt „Talking Trees"; vgl. https://www.waldwachstum.wzw.tum.de/forschung/projekte/talking-trees/ Zugriff 20. Juni 2020.

${ }^{44}$ Vgl. hierzu weiterführend Schneider 2018; zur kritisch-historischen Einordnung dieses Ansatzes in die Kultur- und Mediengeschichte der Mensch-Pflanze Kommunikation Kuni 2020.

45 Vgl. https://blog.zhdk.ch/marcusmaeder u. https://www.marcusmaeder.net sowie exemplarisch das Projekt ,trees“ (zusammen mit dem Ökophysiologen Roman Zweifel), https://www. wsl.ch/de/projekte/trees-1.html Zugriff jeweils 20. Juni 2020.

${ }^{46}$ Vgl. Klaus Littmann: „For Forest. Die ungebrochene Anziehungskraft der Natur“ (2019); temporäre Installation, basierend auf einer Bildidee des Malers Max Peintner, u. hierzu https://www.klauslittmann.com/projekte/for-forest-die-ungebrochene-anzieh ungskraft-der-natur-eine-temporaere-kunstintervention-von-klaus-littmann-2019 Zugriff 20. Juni 2020.

${ }^{47}$ Vgl. „Tempelhofer Wald“ (2019), https://www.tempelhoferwald.berlin/ Zugriff 20. Juni 2020. Verweisen lässt sich für den Großraum Rhein-Main in diesem Kontext auch auf das seit 2002 aktive Projekt „Waldkunst e. V.“, das unter der Leitung der Kulturanthropologin Ute Ritschel „Waldkunst-Lehrpfade“, Ausstellungen, Symposien und weitere Aktivitäten von
} 
als konstruktive Beiträge zu einer angewandten, sozial-ökologisch orientierten Nachhaltigkeitsforschung betrachten.

Für eine Erkenntnis allerdings wird es kaum noch umfangreicherer Studien bedürfen: Allein mit maßvoller Nutzung und Nachpflanzen beziehungsweise Wiederaufforsten - also Maßnahmen, wie sie Hans Carl von Carlowitz seinerzeit zuvorderst im Auge hatte - werden wir unsere Wälder und unsere Baumbestände kaum erhalten können. Und auch eine weitere Erkenntnis scheint sich mittlerweile auch jenseits der bemessenen Kreise im weitesten Sinne professionell mit der Materie befasster Menschen - ob es sich nun um Forstwissenschaftler*innen oder Umweltwissenschaftler*innen, in der Forstwirtschaft, in der Landschaftsgärtnerei oder im Umweltbereich Tätige, um im Feld engagierte Aktivist*innen oder zum Thema arbeitende Künstler*innen handelt - zunehmend durchzusetzen: Baumbestände, sei es nun im Stadtgrün oder in Form von Wäldern, spielen für den Erhalt und die Förderung unserer auf vielfältige Weise bedrohten Artenvielfalt sowie sehr konkret dafür, dass die Spezies Mensch auch weiterhin und auch perspektivisch unter ihr zuträglichen Konditionen diesen Planeten besiedeln kann, eine wichtige Rolle.

Künstlerische Projekte - und die Bilder, die sie kommunizieren - tragen dazu bei, die Bedeutung dieses Komplexes und die Dringlichkeit eines gesamtgesellschaftlichen Handlungsbedarfs, über den angesichts eines zunehmend beschleunigten Baum- und Waldsterbens eigentlich kaum Zweifel bestehen sollten, weiter ins Bewusstsein zu rücken. ${ }^{48} \mathrm{Um}$ es dort auf breiterer Basis nachhaltig zu verankern, wird es eines Mittuns auf vielen Ebenen bedürfen. ${ }^{49}$

und mit Künstlerinnen und Künstlern ausrichtet, die auf ihre Weise - und oftmals auch programmatisch auf Nachhaltigkeitshandeln ausgehend - zur Kommunikation der Bedeutung des Lebensraums Wald beitragen; vgl. https://www.waldkunst.com/ Zugriff 20. Juni 2020.

48 Von der Wahrnehmung dieser Dringlichkeit zeugen neben den bereits genannten Projekten auf ihre Weise auch die zahlreichen populärwissenschaftlichen Publikationen, die in jüngerer Zeit zum Thema Wald und Bäume erschienen sind; im engeren Feld der Kunst lässt sich auf Ausstellungsprojekte wie „Nous les arbres/Trees“ (Fondation Cartier, 2019) oder „Among the Trees" (Hayward Gallery/The Southbank Centre, London, 2020), aber auch das Thema in einem weiteren Radius einbettende Projekte wie „Critical Zones“ (ZKM Karlsruhe, 2020) verweisen; vgl. Pelletier und Couton 2019 sowie zu „Critical Zones“ s. https://zkm.de/de/aus stellung/2020/05/critical-zones und https://critical-zones.zkm.de/\#!/Zugriff 20. Juni 2020.

${ }^{49}$ Etwa auch deshalb, weil davon auszugehen ist, dass hier weitere sozial-ökologische Faktoren eine wesentliche Rolle spielen; die für US-amerikanische Abholzungskampagnen engagierten Waldarbeiter etwa, die mit ihrer Arbeit für sich und ihre Familien die Existenz sichern müssen, werden nicht unbedingt zum Leserkreis von Büchern wie Richard Powers “ „Die Wurzeln des Lebens“ (,The Overstory“) gehören, in denen sie eine tragende Rolle spielen (siehe Powers 2018), und/oder das Privileg genießen, sich im Rahmen des Besuchs von Ausstellungen mit den „Critical Zones“ unseres Planeten zu befassen. 


\section{Summ, summ: Die Bienen}

Greift man insbesondere letztere Perspektive auf, so lässt sich neben den Bäumen eine Reihe weiterer Lebewesen benennen, die man gleichsam als ,Zeiger'Organismen und zugleich ,Medien' einer populären Kommunikation ökologischen Bewusstseins bezeichnen könnte. Unter diesen wiederum dürfte namentlich in unseren Breiten vielen anderen, je auf ihre Weise ebenfalls populären Vertreter*innen aus dem Pflanzen- und Tierreich wie dem Edelweiß, der Kornblume, dem Wolf, verschiedenen Singvögeln sowie in jüngerer Zeit auch Schmetterlingen insbesondere ein Insekt den vorgenannten unschwer den Rang ablaufen - und zwar auch aufgrund seines markanten Eintrags in die Kulturgeschichte: die Honigbiene. ${ }^{50}$ Dies wiederum schließt, wie im Folgenden noch zu zeigen sein wird, auch ihre Rolle in der Nachhaltigkeitsdebatte ein. Ihren herausgehobenen Status speziell in diesem Kontext verdankt die Honigbiene wohl nicht zuletzt der auch für Laien leicht nachvollziehbaren Einsicht, dass zahlreiche für die menschliche Ernährung wichtige Nutzpflanzen für die Fruchtbildung - und prinzipiell auch die Fortpflanzung ${ }^{51}$ - auf eine Bestäubung durch spezifische Insektenarten angewiesen sind, unter denen wiederum sich die Honigbiene wohl der größten populären Prominenz erfreut. Zugleich werden von der auf einen massiven Einsatz von Pestiziden und Herbiziden sowie großflächigen Monokultur-Anbau setzenden Landwirtschaft Insekten ihrer Lebensgrundlagen beraubt, unabhängig davon, ob sie Menschen als so genannte „Schädlinge“ oder „Nützlinge“ gelten. Beides hat sich inzwischen auch über den engeren Radius ökologisch Engagierter hinaus herumgesprochen. So ist gerade in den letzten Jahren, in denen regelmäBig in der Tagespresse und anderen Nachrichtenmedien alarmierende Meldungen zu weltweit auftretendem Bienensterben kursieren, immer wieder das Albert Einstein zugeschriebene Zitat zu lesen: „Wenn die Biene einmal von der Erde verschwindet, hat der Mensch nur noch vier Jahre zu leben. Keine Bienen mehr, keine Bestäubung mehr, keine Pflanzen mehr, keine Tiere mehr, keine Menschen mehr. “52

\footnotetext{
${ }^{50}$ Hiervon zeugt unter anderem auch eine beeindruckende Anzahl von kultur- und populärwissenschaftlichen Publikationen zu Bienen, insbesondere zu Honigbienen, die in den vergangenen Jahren erschienen sind und auf die hier näher einzugehen den gegebenen Rahmen sprengen würde. Für eine informative Einführung vgl. Tautz 2007; zu ausgewählten Perspektiven vgl. weiterführend unten.

${ }^{51}$ Dies wiederum gilt für viele Nutzpflanzen nur eingeschränkt, insofern hier traditionell Züchtungs- und Veredelungsverfahren sowie seit einigen Jahrzehnten auch Gentechnik zum Einsatz kommen.

${ }^{52}$ Vgl. für eine quellenreiche Rekonstruktion der Zuschreibung Freistetter 2015.
} 
Wiewohl diese Aussage, unabhängig von ihrer Provenienz, kaum beim Wort zu nehmen ist, haben die Schreckensnachrichten vom Verschwinden der Bienen eine erhebliche Öffentlichkeitswirksamkeit entfaltet, wie sie vielen anderen bedrohten Tier- und Insektenarten bislang leider weitgehend versagt geblieben ist. ${ }^{53}$ Denn anders als andere Bestäuber*innen kann die Honigbiene, als Nutztier sowie wiederum nicht zuletzt aufgrund ihrer historisch verbrieften positiven kulturellen Rezeption, auf breiter Basis mit Sympathien rechnen. ${ }^{54}$ Wenngleich kaum von einer breitenwirksamen Bekanntheit der Kulturgeschichte als solcher auszugehen ist $^{55}$, so gehören doch deren populäre Ausläufer - etwa, noch vor Waldemar Bonsels ,Immenmärchen“ selbst, die „Biene Maja“ der ihre Vorlage recht frei umsetzenden Zeichentrickserie ${ }^{56}$ - sowie eben ganz grundsätzlich das Bild der fleißigen, uns mit köstlicher Süße versorgenden Honigbiene zum Allgemeingut.

Vor diesem Hintergrund verwundert es nicht, dass die Biene in den vergangenen Jahren $\mathrm{zu}$ einer prominenten „Botschafterin für nachhaltiges Handeln“57 geworden ist und allenthalben Projekte entstanden sind, in denen Bienen eine zentrale Rolle spielen - und zwar allem voran im urbanen Raum. Tatsächlich sind es gerade die Städter*innen, die das Imkern zunehmend für sich entdecken und damit dem zuvor noch von Nachwuchssorgen geplagten Handwerk bereits zu

${ }^{53}$ Indessen ist das Forschungsfeld als solches längst nicht mehr allein auf die Naturwissenschaften beschränkt; so haben sich inzwischen in den Kulturwissenschaften die Extinction Studies etabliert - und auch in der künstlerischen Forschung ist das Artensterben zu einem wichtigen Thema auch über populäre Spezies hinaus geworden.

54 Ähnliches gilt auch in anderen Zusammenhängen als dem hier angesprochenen Bestäubungskontext, in dem u. a. aufgrund von Spezialisierungen in Artengemeinschaften eine Vielfalt unterschiedlichster Insekten eine wichtige Rolle spielt. So stehen in Deutschland zwar prinzipiell alle Staaten bildenden Insekten unter Artenschutz; Hornissen, Wespen und Ameisen wird jedoch im Einzelfall keineswegs so freundlich begegnet; Wildbienen flogen ebenfalls lange unter dem Radar.

${ }^{55}$ Für einen allgemeinverständlichen, fundierten Überblick vgl. Dutli 2012; für weiterführende Literatur zu spezifischen Perspektiven vgl. unten.

${ }^{56} \mathrm{Vgl}$. Bonsels 1912; die als deutsch-japanische Koproduktion entstandene Zeichentrickserie „Die Biene Maja“ (J/D/AT, 1975-1979) erhielt 2013 eine Neufassung in 3-D (D/F 20132017). Direkt mit der (Zeichentrick-),Biene Maja“ bewerben sowohl der BUND seine auf Kinder zugeschnittenen Bildungsmaterialien zum Thema „Bienen und Pflanzen“, https://www. bund.net/themen/tiere-pflanzen/wildbienen/wildbienen-helfen/umweltbildung/ - als auch die Supermarktkette REWE ihre mit dem NABU verfolgte(n) Insektenschutz-Initiative(n), https:// www.rewe.de/nachhaltigkeit/unsere-ziele/projekte/zuhause-fuer-insekten/Zugriff jeweils 20. Juni 2020.

57 Vgl. das Motto des „Bienenretter“-Bildungsprojekts, https://www.bienenretter.de/ Zugriff 20. Juni 2020. 
einer unverhofften Renaissance verholfen haben. ${ }^{58}$ Zumal diese urbane Imkerei in der Regel nicht gewerblich betrieben wird, ist sie aus ökologischer Perspektive prinzipiell in mehrfacher Hinsicht ein Zugewinn. Zwar können Stadtbienen die durch Monokulturen und industriell betriebene Landwirtschaft mit bedingte Verdrängung der Honigbiene und deren Konsequenzen für das Ökosystem nicht kompensieren - und es gibt inzwischen durchaus auch kritische Stimmen, die vor den Folgen einer urbanen ,Honigbienen-Monokultur' warnen. ${ }^{59}$ Gleichwohl tragen die Bienen in den Städten, wo sie in Parks, auf Friedhöfen, in Gärten und Brachen reiche Nahrung finden, als Bestäuberinnen zum Erhalt der Artenvielfalt bei. Honig von Stadtbienen ist - anders als im Stadtraum angebautes Obst und Gemüse und auch anders als so mancher auf dem von industrieller Landwirtschaft geprägten Land geimkerte Honig - kaum schadstoffbelastet und obendrein als lokales Produkt weitaus verträglicher als ein Gutteil des aus unterschiedlichen Quellen stammenden Honigs, der im Supermarkt zum Verkauf angeboten wird. ${ }^{60}$ Und schaut man auf dessen für ein von Tieren durchaus mühevoll hergestelltes Naturprodukt beschämend niedrige Preise, die sich dem globalen Markt und dessen von Handels- und Preispolitiken gesteuerten Produktionsbedingungen verdanken, so kommt ein weiterer Aspekt hinzu: Wer selbst als Imker*in über mehrere Monate des Jahres mit der Hege und Pflege von Bienenvölkern befasst ist, aus nächster Nähe beobachten kann, wie diese Honig produzieren, und wer ihn selbst den Stöcken entnimmt und in Gläser füllt, weiß den Wert des Guts ganz anders zu schätzen. ${ }^{61}$

${ }^{58}$ So bieten neben und teils in Zusammenarbeit mit den traditionellen Imkervereinen inzwischen auch zahlreiche der neu entstandenen Initiativen Einführungen in die Imkerei an; vgl. exemplarisch die Angebote von „Stadtbienen“, https://www.stadtbienen.org/ Zugriff 20. Juni 2020; in den vergangenen Jahren sind nicht nur in Deutschland allenthalben „Stadtimkereien“ entstanden - insgesamt kann man von einem regelrechten Boom sprechen. Zur Stadtimkerei in sozial-ökologischer Perspektive vgl. weiterführend Kosut und Moore 2013.

${ }^{59}$ Vgl. für eine kompakte kritische Reflexion zum Stadtimkerei-Boom Berger 2019; zur Verschärfung der Problematik aufgrund eines durch urbane Imkerei erhöhten Drucks auf andere Insekten (und damit auch die Artengemeinschaften, für deren Erhaltung diese eine Rolle spielen) weiterführend den auch von Berger referierten Beitrag von Ropars et al. 2019, die wiederum weitere kritische Studien anführen.

${ }^{60}$ Honig trägt durch die enthaltenen Pollen einen, lokalen Stempel ‘; Pollen, für die keine generelle Expositionsgewöhnung besteht, können leichter allergische Reaktionen hervorrufen und sind insofern eher ein Problem als etwa eher als durch Lebensmittelkontrollen detektierbare (Pflanzen-)Toxine und Schadstoffe.

${ }^{61}$ Vgl. zu Honig allgemein Horn und Lüllmann 2006; Honigproduktion und -konsum sind insofern ein auf den Nägeln brennendes Nachhaltigkeitsthema, da in der globalen Wirtschaft eine industriell organisierte Bienenzucht und Honig-Gewinnung mit hoch problematischen Konsequenzen dominiert - die ihrerseits inzwischen nicht nur ebenfalls mit 


\section{$5 \quad$ Das soziale Leben der Stadtbienen}

Dass die Frankfurter Künstlergruppe „finger“ in Deutschland zu den Pionier*innen der urbanen Imkerei gehört, ist kein Zufall. Seit ihrem Zusammenschluss im Jahr 1998 haben sich die an ,finger“" beteiligten Künstler*innen ${ }^{62}$ in zahlreichen Projekten intensiv mit den Zusammenhängen von Ökonomie, Ökologie und Gemeinschaftlichkeit beschäftigt. In diesem Kontext ist auch die 2007 erfolgte Gründung der „Stadtimkerei“ durch die „finger“-Künstler Florian Haas und Andreas Wolf zu sehen, bei der es nicht allein um Honigproduktion geht. Im Mittelpunkt stehen vielmehr die Bienen selbst - genauer gesagt, wie es die beiden Stadtimker auf ihrer Webseite formulieren, deren „Lebenswelt“, die in den Projekten ,in Analogie zu aktuellen gesellschaftlichen Entwicklungen und Themen" gesetzt wird. Der Bienenstock bzw. -staat dient dabei als Modellorganismus und „Material“ für die „Kunstbeiträge zu Umwelt- und Gesellschaftsprozessen“, die Haas und Wolf in verschiedenen Formaten für unterschiedliche Kontexte entwickeln. ${ }^{63}$

So fanden die ersten Bienenstöcke auf Einladung von Gerald Hintze, der im Auftrag der evangelischen Diakonie im Frankfurter Bahnhofsviertel als Kurator der ebendort gelegenen Weißfrauenkirche Kunstprojekte mit sozialem Bezug organisierte, im Kirchturm Aufstellung; ursprünglich war geplant gewesen, die Aktivitäten der Stadtimkerei mit der ebenfalls von der Diakonie betriebenen Anlaufstelle für Obdachlose, „Weser 5“, zu verknüpfen. ${ }^{64}$ Vor diesem Hintergrund riefen die beiden Künstler die „Gemischte Bienengruppe“ ins Leben, in

Umweltbelastungen durch Toxine, sondern auch mit Folgen ihrer eigenen Strukturen zu ringen hat.

${ }^{62} \mathrm{Zu}$ den Gründungsmitgliedern 1998 zählten Martin Schmidl, Florian Haas, Martin Brandt und Andreas Wolf; zeitweise waren weitere Akteur*innen beteiligt. Die ehemalige Homepage der Gruppe finger ist inzwischen zur Homepage der Stadtimkerei finger geworden; die dieser voran gegangenen Projekte sind im Projektarchiv aufgeführt; vgl. https://www.fingerweb.org/ und https://archiv.fingerweb.org/html/projekte.html Zugriff jeweils 20. Juni 2020.

${ }^{63}$ Vgl. den einführenden Text auf der Startseite der Homepage, https://www.fingerweb.org/, Zugriff 20. Juni 2020. Der Begriff „Modellorganismus“ scheint dabei - auch mit Blick auf die Kulturgeschichte, in welcher der Bienenstaat als Metapher, Modell sowie als Utopie für die menschliche Gesellschaft begegnet - passender als der von den Künstlern selbst in Anführungszeichen gesetzte Begriff des Materials; vgl. hierzu weiterführend unten.

${ }^{64}$ Vgl. https://www.fingerweb.org/projekte-2007/irgend-etwas-bl\%C3\%BCht-immer.html Zugriff 20. Juni 2020. Für ,Weser 5“ hatte Hintze (1949-2012) während seiner Wirkungszeit regelmäßig Künstler*innen engagiert. Zu Hintzes beispielgebender Arbeit vgl. Kaufmann 2015. 
der sich Menschen aus unterschiedlichen sozialen Schichten gemeinsam um Bienenstöcke kümmern. ${ }^{65}$ Als die Kirche 2008 renoviert werden musste, migrierten die Stöcke aufs Dach des Museums für Moderne Kunst (MMK), wo Haas und Wolf seither regelmäßig auch öffentliche Veranstaltungen wie Kinder-Workshops und ein „Honigfrühstück“ anboten; 2019 zogen die Stöcke ans andere Mainufer auf das Museum Angewandte Kunst um. ${ }^{66}$ Zeitgleich mit dem ersten Umzug der Stöcke erhielt die „Gemischte Bienengruppe“ für mehrere Jahre einen eigenen Ort mit Bienenstöcken auf einem ehemaligen Campingplatz am südlichen Mainufer beim Niederräder „Licht- und Luftbad“, das ehedem insbesondere für die Frankfurter Arbeiterschicht einen beliebten Erholungsraum darstellte. ${ }^{67}$

Nachdem der kleine Kunstraum, den die Stadtimkerei in direkter Nachfolge der ursprünglichen ,finger“-Aktivitäten im Zentrum der Frankfurter Innenstadt als Büro mit Ausstellungen und Veranstaltungsprogramm betrieb, aus Kostengründen nicht länger zu halten und zudem der Bienenstand der „Gemischten Bienengruppe" am Mainufer einer Brandstiftung zum Opfer gefallen war, entschlossen sich Haas und Wolf zum Umzug an einen auf der lokalen Landkarte erfolgreicher ökologischer Transformationen eingetragenen Ort: den im Frankfurter Grüngürtel zwischen Bonames und Kalbach-Riedberg gelegenen „Alten Flugplatz“, einen ehemaligen US-amerikanischen Militärflughafen, der ab 2002 renaturiert und in ein Natur- und Freizeitgelände umgestaltet wurde. ${ }^{68}$ In ihren Installationen und Aktionen sowie begleitenden Veranstaltungen vermitteln die Künstler Kindern und Erwachsenen nicht nur Grundlagenwissen über die Imkerei, sondern erkunden gemeinsam mit ihnen auch die kulturgeschichtlichen und ästhetischen Dimensionen des Bildes vom „Bienenstock“.

Gerade Letztere sind hierbei ebenso wie in den zahlreichen weiteren Aktionen und Projekten, die von den beiden Künstlern in den vergangenen Jahren im

\footnotetext{
${ }^{65} \mathrm{Vgl}$. https://www.fingerweb.org/projekte-2008/die-gemischte-bienengruppe.html Zugriff 20. Juni 2020.

${ }^{66} \mathrm{Zu}$ den Bienenstöcken auf dem Dach des MMK Frankfurt vgl. https://www.fingerweb.org/ projekte-2009/das-mueum-ein-bienenkorb.html Zugriff 20. Juni 2020 (die den Museumsbienen gewidmeten Seiten auf der Homepage des MMK gibt es nicht mehr); zum neuen Standort im Museum Angewandte Kunst vgl. https:/www.museumangewandtekunst.de/de/ vermittlung/neues-museum-fuer-bienen/ Zugriff 20. Juni 2020.

${ }^{67} \mathrm{Vgl}$. https://www.lilu-frankfurt.de/, Zugriff 20. Juni 2020, sowie zur auch unter sozialökologischen Gesichtspunkten interessanten historischen Badekultur in Frankfurt Rödel 2013. ${ }^{68}$ Vgl. zum „Alten Flugplatz“ als Natur-Lernstation im Grüngürtel die Webseite der Stadt Frankfurt, https://frankfurt.de/themen/umwelt-und-gruen/orte/gruenguertel/ziele/intere ssante_orte/alter_flugplatz Zugriff 20. Juni 2020; zur Geschichte und Umgestaltung des Ortes Förster 2010.
} 
Rahmen von Ausstellungen in Kunstinstitutionen und im öffentlichen Raum entwickelt wurden, durchaus entscheidend. Für jeden Ort, jeden Anlass entwickeln Haas und Wolf ein auf diesen zugeschnittenes künstlerisches Gestaltungskonzept, das mit ihrer Grundidee einer wechselseitigen Erhellung der Perspektiven korrespondiert; dies umfasst alle Elemente des jeweiligen Projekts, vom Ausstellungsraum bis hin zu Informationsmaterialien, vom Honigglas bis zu unterschiedlichen Konstruktionen, in denen Bienenstöcke beziehungsweise Beuten untergebracht werden können. So ist beispielsweise das als mobile, modular aufgebaute Konstruktion konzipierte „Frankfurter Bienenhaus“ (2014) einerseits auf die Bedürfnisse der Imkerei zugeschnitten, der ein an verschiedene Orte verbringbarer Unterstand für temporär zu Bienenweiden verbrachte Stöcke zupass kommt. Andererseits bietet es aufgrund seiner mit Bildern, Texten und Mustern versehenen Wände den Menschen eine ästhetische „Augenweide“ und lädt zum Nachdenken über Insekten- und Menschengesellschaften ein. ${ }^{69}$ Ganz ähnlich funktioniert auch das „Neue Museum für Bienen“ (seit 2011), das die alte Tradition der „Figurenbeute“ als bildhafter Gestaltung des Bienenstocks aufnimmt ${ }^{70}$ und in eine Modell-Architektur transformiert, die zugleich an die Institution des (Kunst-)Museums als vitalem Gedächtnis- und Denkraum anknüpft. Seine auf die Stöcke gesetzten Schauhäuser können dabei je nach Ausstellungskontext neu eingerichtet werden - wie etwa 2013, als das Museum in der Kunsthalle Budapest gastierte. Haas und Wolf nahmen die seinerzeit in und um Ungarn entbrannten Diskussionen um die demokratische Orientierung der Regierung Orban auf, indem sie das Thema „Demokratie“ in den Mittelpunkt der auf drei Räume beziehungsweise Bienenstöcke verteilten Museumsschau stellten. Während der erste Raum danach fragte, ob im Bienenstaat eine ,bessere“ Demokratie herrsche, lud der zweite mit einer Präsentation über Insekten als Nahrungsalternative zum in Europa traditionell auf Säugetiere, Geflügel und Fische fokussierten Fleischkonsum dazu ein, demokratisch über die Frage der menschlichen Ernährung

\footnotetext{
${ }^{69} \mathrm{Vgl}$. https://www.fingerweb.org/projekte-2014/das-frankfurter-bienenhaus-in-dortmund. html Zugriff 20. Juni 2020; seinen Namen verdankt das mobil konzipierte Bienenhaus nicht dem Standort, sondern der 1926 für Ernst Mays soziale Wohnprojekte des „Neuen Frankfurt“ entworfenen „Frankfurter Küche“ von Margarete Schütte-Lihotzky.

${ }^{70}$ Vgl. zu Figurenbeuten Jung-Hoffmann 1993; für eine zeitgenössische Wiederaufnahme die Figurenbeuten der Bildhauerin Birgit Maria Jönsson; https://www.figurenbeuten.de/ u. https://www.bienenimbauch.de/ Zugriff jeweils 20. Juni 2020.
} 
abzustimmen. Der dritte Raum forderte dazu auf, einer lokalen „Gemischte Bienengruppe" beizutreten und auf diese Weise in der gemeinschaftlich betriebenen Imkerei nachhaltig sozial und ökologisch tätig zu werden. ${ }^{71}$

Wie die Projekte belegen, werden die Bienen von Haas und Wolf nicht als „Natur" repräsentierende, lebende Objekte in den Kontext der Kunst gerückt, sondern vielmehr als Ko-Produzent*innen verstanden, die dazu beitragen, den Menschen neue Perspektiven auf sich, ihre Umwelt und ihre Lebensverhältnisse zu eröffnen. ${ }^{72}$ Ähnliches gilt für die in Anspruch genommene Analogie zwischen Bienenstaat und Gesellschaft, zumal diese nicht länger allein auf den Menschen ausgerichtet bleibt, sondern - wie im „Neuen Lorscher Bienensegen“ sogar wortwörtlich demonstriert ${ }^{73}$ - auch den Bienen selbst eine Stimme gibt: Stets steht die künstlerische Arbeit der Stadtimker im Dienst einer Reflexion der Mensch-TierBeziehung, die das Tierwohl im Auge behält - und gerade deshalb auch in dem, was sie speziell von Mensch zu Mensch vermitteln will, in doppeltem Wortsinn nachhaltig wirksam werden kann. Dies wiederum stellt durchaus eine besondere Qualität ihrer Projekte dar, gerade auch wenn man sie im weiteren Feld der Kunst betrachtet.

Tatsächlich sind die Frankfurter Stadtimker längst nicht die einzigen unter den zeitgenössischen Künstler*innen, die sich für Bienen interessieren und diese in ihre Arbeit mit einbeziehen. Gerade in den vergangenen Dekaden, in denen auch die urbane Imkerei an Fahrt aufgenommen hat, lässt sich hier eine regelrechte Konjunktur verzeichnen. Das Spektrum reicht dabei von unterschiedlichen Verknüpfungen zwischen Imkerei und $\mathrm{Kunst}^{74}$ bis hin zu komplexen künstlerischen Forschungsprojekten wie sie etwa die Belgierin AnneMarie Maes betreibt, die mit

\footnotetext{
${ }^{71} \mathrm{Vgl}$. https://www.fingerweb.org/projekte-2013/neues-museum-fuer-bienen-budapest.html Zugriff 20. Juni 2020.

72 Dementsprechend kann Letzteres sowohl auf die Bienen als auch auf die Menschen bezogen werden.

73 Der „Lorscher Bienensegen“ ist ein aus dem 10. Jahrhundert stammendes Spruch-Notat in einer Handschrift, das Bienen anruft, um sie zur Rückkehr in den Stock zu bewegen. Die Installation mit Bienenstock und vorgelagerter Bildwand (2012) zeigt auf Letzterer eine Neudichtung, in der die Bienen selbst die Konditionen für ihre Rückkehr formulieren; vgl. https://www.fingerweb.org/projekte-2012/neuer-lorscher-bienensegen.html Zugriff 20. Juni 2020.

${ }^{74}$ Die Zahl der Künstler*innen, die sich in diesem Feld engagieren, ist inzwischen Legion; ähnlich wie Haas und Wolf seit langen Jahren ebenso konsequent wie kreativ entwickelt die Österreicherin Christina Stadlbauer ihre Projekte; darunter auch im Rahmen einer Kooperation mit dem Nachhaltigkeits-Bildungsprojekt „Sustainicum“; seit 2012 arbeitet sie gemeinsam mit einem internationalen Team in Finnland; vgl. https://melliferopolis.net/ sowie https:// www.sustainicum.at Zugriff jeweils 20. Juni 2020.
} 
anderen Künstler*innen kooperiert und dabei neue Technologien miteinbezieht, um etwa die Flugrouten von Bienen im Stadtraum zu kartieren, die Klänge von Bienenstöcken zu belauschen oder ,smarte Bienenkörbe“ zu entwerfen. ${ }^{75}$ Andere wiederum setzen auf die Fähigkeiten der Bienen, vorgefundene Strukturen zum Wabenbau nutzen, um Plastiken und Objekte zu kreieren. ${ }^{76}$

So sehr die Ergebnisse dieser ,Zusammenarbeit“ ästhetisch faszinieren und die in jüngerer Zeit in den Human-Animal-Studies sowie den Multispecies Studies virulenten Diskussionen darüber weiter befeuern mögen, ob und inwiefern den Tieren künstlerische Kompetenzen zugestanden werden können ${ }^{77}$ : Ob entsprechende Objekte und deren unter Netzkonditionen oftmals auch jenseits des Radius der Kunst verbreitete Bilder über die temporäre Bewunderung hinaus dazu beitragen können, Tierrechte und Tierwohl dauerhaft in den menschlichen Horizont zu rücken, dürfte vorerst offen bleiben. ${ }^{78}$ Was sie indessen in jedem Fall belegen ist, dass sich mit den Kompetenzen der Kunst und ihren auf die ästhetische Anschauung ausgerichteten Mitteln gesellschaftliche Aufmerksamkeit generieren und einer Sensibilität für die Komplexität der Zusammenhänge zuarbeiten lässt, in denen Mensch-Tier-Verhältnisse immer schon gestanden haben - die insbesondere jedoch dann interessieren müssen, wenn es um Nachhaltigkeitsperspektiven geht. $^{79}$

${ }^{75}$ Vgl. für weiterführende Informationen zu den Projekten die Webseite der Künstlerin, https://annemariemaes.net/ Zugriff 20. Juni 2020. Das erste Bienenvolk hatte Christina Stadlbauer 2009 zu Maes gebracht, die zu dieser Zeit im Kunstraum OKNO engagiert war, auf dessen Dachterrasse Bienenstöcke im Rahmen des Projekts ,open_green“ Aufstellung fanden.

76 So z. B. die Kanadierin Aganetha Dyck (https://www.aganethadyck.ca/), der Slovake Tomáš Gabzdil Libertíny (https://www.tomaslibertiny.com), die Französin Luce Moreau oder der US-Amerikaner Garnett Puett (https://www.jackshainman.com/artists/garnett-puett/) Zugriff jeweils 20. Juni 2020.

77 Vgl. hierzu den Abschnitt „Animalische Ästhetik“ in Ullrich 2016, 211-212 sowie speziell zu Bienen Kosut und Moore 2014; zu den Multispecies Studies exemplarisch Kirksey 2014 und zu Bienen die Artikel „Buzz“, „Domestication“, „Swarm“ und „Swarming“ auf der Webseite des Multispecies Salon, vgl. https://www.multispecies-salon.org/?s=bees Zugriff 20. Juni 2020; zur Honigbiene in Multispezies-Perspektive des Weiteren neben Kosut und Moore 2013 auch Luttrell 2017.

${ }^{78}$ Dies zumal stets zu fragen ist, ob überhaupt und wenn ja, unter welchen Konditionen, für welche Zwecke und zu welchen Enden sich ein Lebewesen ein anderes (oder, im Plural: andere) zu eigen machen darf.

${ }^{79}$ In diesem Kontext sind, neben den eingangs bereits referenzierten Titeln (Goehler 2010, Kagan 2011), die kritischen Überlegungen von T. J. Demos erhellend, vgl. Demos 2016, insbesondere Kap. 1, The Art and Politics of Sustainability, 31-62. 


\section{$6 \quad$ Hive Mind und Honigpumpe}

Deren Reflexion anzuregen, mag man in der Tat als eine Aufgabe der Kunst und namentlich einer Kunst, die sich als ,ars longa“ verstehen will - betrachten. Doch wenngleich ästhetische Bildung im weitesten Sinne ebenso wie die Auseinandersetzung mit Kunst hier einen wichtigen Beitrag leisten kann, wäre es wohl verfehlt, Künstler*innen an erster Stelle auf eine solche Perspektive zu verpflichten. Allerdings kann nicht zuletzt die für viele zeitgenössische Positionen charakteristische Deutungsoffenheit und Unabgeschlossenheit dazu einladen, in der Auseinandersetzung mit den Arbeiten selbst entsprechende Perspektiven zu erschließen - und Letzteres wiederum wird sich umso mehr anbieten, wenn diese bereits über den jeweiligen Präsentationskontext angelegt sind.

Wer etwa Pierre Huyghes komplexer Installation „Untilled“ (2011-12) in jüngerer Zeit in einer Museumsausstellung begegnet, dürfte ohne Vorwissen um den ursprünglichen Entstehungszusammenhang der Arbeit beim Anblick des liegenden Aktes, dessen Kopf komplett von einer Bienenwabe umkleidet ist, nicht zwangsläufig an Ökologie oder gar Nachhaltigkeit denken. ${ }^{80}$ Entwickelt hat der Künstler das Projekt jedoch ursprünglich für die documenta 13, deren Kuratorin Carolyn Christov-Bakargiev der Auseinandersetzung mit „NaturKulturen“"81, einschließlich der mit dieser verknüpften ökologischen und sozialen Fragen, im Konzept der Schau einen denkbar zentralen Platz eingeräumt hatte. ${ }^{82}$ Huyghe gestaltete für „Untilled“ eine Brache am Rande der Kasseler Karlsaue so um, dass zum Zeitpunkt der Eröffnung der Großausstellung nurmehr zu erahnen war, welche Elemente und Partien der Landschaft neu angelegt und welche so wie vorgefunden belassen waren. Folgte man den Wegen zwischen Wildwuchs und neu aufgeschütteten, größten Teils begrünten Hügeln, konnte man dabei nicht nur auf den liegenden Akt mit seinem von Bienen umsummtem Waben-Kopf und auf einen durchs Grün streifenden, weißen Windhund mit einem in leuchtendem rosa gefärbten Lauf stoßen. Sondern auch auf den liegenden Baumriesen einer toten

\footnotetext{
${ }^{80}$ Vgl. für einen Überblick über Huyghes Werk Lavigne et al. 2014; zu „Untilled“ ebd., S. 186-198.

${ }^{81}$ Der Begriff „NatureCultures“ wurde von Donna Haraway eingeführt, deren Position für Christov-Bakargiev einen wichtigen Bezuspunkt darstellte; vgl. Haraway 2003 und Haraway 2008 sowie weiterführend Haraway 2016. Haraway war Vortragsgast in Kassel und steuerte auch einen Text für die Publikationsreihe „100 Notes - 100 Thoughts“ bei.

${ }^{82}$ Vgl. die Dokumentationen und Publikationen zur documenta 13 (2012) sowie insbesondere das alle Einzelpublikationen der „100 Notes - 100 Thoughts“ zusammenfassende „Book of Books“ (Sauerländer 2012), in dem neben das kuratorische Konzept erläuternden Beiträgen u. a. auch eine ,reading list“ enthalten ist.
} 
Eiche, von der es hieß, es handele sich um einen jener Bäume, die ehedem im Zuge von Beuys" „Stadtverwaldung“ angepflanzt worden waren.

Anders als „7000 Eichen“ war „Untilled“ nicht auf den Verbleib in Kassel hin konzipiert. Dennoch scheint es voreilig, dem Projekt jegliche Nachhaltigkeit abzusprechen. Ebenso wie das zwischen Traum und Wirklichkeit oszillierende, poetische Bild, das es bei jenen hinterlassen konnte, die den Ort während der Laufzeit der documenta 13 aufgesucht haben - wovon nicht zuletzt das Echo zeugt, das die Arbeit im Netz, in der Presse und anderen Publikationen fand -, dürften wohl auch der temporäre Eingriff in das bestehende Biotop mit den Erdaufschüttungen, Saaten und Pflanzungen sowie nicht zuletzt den Bienen, Hunden und Menschen dem Gelände bleibende Spuren eingetragen haben.

Insbesondere in Verbindung mit der toten Eiche mag Huyghes „Hive Mind“ 83 schließlich von der - auch historisch eingetragenen - Distanz und Nähe zur unauslöschlich mit der Kasseler documenta verknüpften Position von Joseph Beuys zeugen. Tatsächlich hatte dieser 1977, mithin fünf Jahre bevor er zur documenta 7 seine Pflanzaktion für „7000 Eichen“ initiierte, mit seiner „Honigpumpe am Arbeitsplatz" das Museum Fridericianum in einen Bienenstock transformiert. Während sich im zentralen Treppenhaus des Gebäudes eine riesige Kupferwalze in einem Margarineberg drehte, flossen $150 \mathrm{~kg}$ Honig durch ein über die Geschosse verteiltes Schlauchsystem. Am „Arbeitsplatz“ selbst versammelte Beuys allerdings keine Bienen, sondern lud zusammen mit Mitstreiter*innen der von ihm begründeten „Free International University“ zu Vorträgen und Diskussionen ein (vgl. Loers und Witzmann 1993, S. 145-220).

Beuys ' Beschäftigung mit dem Bienenstaat beginnt nicht erst im Zuge seines ökologischen Engagements. Bereits um 1950 formt er seine erste „Bienenkönigin“" aus Wachs ${ }^{84}$; zahlreiche frühe Blätter zeugen von seiner intensiven Auseinandersetzung mit dem Thema. Im Anschluss an Rudolf Steiner - dessen anthroposophische Bienenkunde wiederum von historischen wie zeitgenössischen sozialutopischen und politischen Lesarten des Insektenstaats als Metapher für die Organisation menschlicher Gemeinschaft mitgeprägt war - wollte Beuys

\footnotetext{
${ }^{83}$ Wiewohl Huyghe diese Assoziation nicht explizit forciert, steht sie - insofern dies sowohl der Zeit- als auch der Werkkontext nahelegen - im Raum; zum kulturgeschichtlichen Resonanzraum des „Hive Mind“ vgl. Kuni 2010b.

${ }^{84}$ Vgl. Joseph Beuys: „Bienenkönigin I“ (1947-52); zwei 1952 entstandene Fassungen befinden sich heute im „Block Beuys“ im Landesmuseum Darmstadt. Vgl. zu den „Bienenköniginnen“ und Beuys' früher Beschäftigung mit Bienen Veit Loers in Loers und Witzmann 1993, 55-63.
} 
die Biene als „Wärmewesen“ und den Bienenstaat als „sozialen Organismus“ ${ }^{85}$ verstanden wissen. Letzteres wiederum lässt seine Position - und insbesondere seinerzeit mit entsprechenden Aktionen verbundene Werke wie die „Honigpumpe am Arbeitsplatz“ - aus heutiger Sicht ebenso aktuell wie anschlussfähig erscheinen.

Mit Blick auf Projekte wie „Die Honigpumpe am Arbeitsplatz“ und „7000 Eichen" kann man Beuys wohl mit Fug und Recht als Pionier auf dem Feld einer „Kunst der Nachhaltigkeit“ bezeichnen. Nachhaltig wirken nicht zuletzt die Denkbilder fort, die er mit seinem Werk hinterlassen hat. Indes hat eine jüngere Generation zeitgenössischer Künstler*innen längst damit begonnen, eigene Zugänge und Perspektiven zu entwickeln. Dabei können sie auf bereits angelegte Fundamente bauen. Beuys galt seinerzeit noch als Provokateur und seine „Erweiterung des Kunstbegriffs“ in die Gesellschaft, in die Auseinandersetzung mit Ökologie und Ökonomie hinein Vielen als Affront. ${ }^{86}$ Heute hingegen scheint es nahezu selbstverständlich, dass Künstler*innen nicht allein aus dem Atelier heraus operieren, sondern mit Projekten direkt in die Öffentlichkeit gehen - und dabei etwa auch unter den Vorzeichen der Kunst Bäume pflanzen oder eine Stadtimkerei betreiben. Kunst, die kulturelle Bildung, ökologisches und soziales Engagement verknüpft, hat sich als zukunftsfähig erwiesen: Als „,ars longa“, die das Thema Nachhaltigkeit - und die mit ihm verknüpften Fragen und Komplexe - nicht nur aufgreift und in Bilder fasst, sondern direkt zum Handeln und Mittun einlädt.

\section{Literatur}

Berger, R. 2019. Irrweg Stadtimkerei, das sogenannte Bienensterben. Telepolis 17.11.2019 https://www.heise.de/tp/features/Irrweg-Stadtimkerei-das-sogenannte-Bienensterben4586068.html. Zugegriffen: 20. Juni 2020.

Beuys, J. 1978. Aufruf zur Alternative. Frankfurter Rundschau 288: (23.12.1978), 2.

Böhme, G. 1989. Für eine ökologische Naturästhetik. Frankfurt a. M.: Suhrkamp.

Bonsels, W. 1912. Die Biene Maja und ihre Abenteuer. Ein Roman für Kinder. Berlin: Schuster und Löffler.

${ }^{85}$ Der Begriff ,,sozialer Organismus“ begegnet zunächst in der als Disziplin noch im Entstehen begriffenen Soziologie des 19. Jahrhunderts; heute prominent insbesondere in der Anthroposophie, insofern er auch von Rudolf Steiner verwendet wurde; vermittelt durch Joseph Beuys, der Steiner intensiv rezipiert hat, erlangte er u. a. auch im Diskurs um gesellschaftlich engagierte Kunst einige Prominenz. Vgl. zur Organismus-Metaphorik Schlechtriemen 2008; zu Beuys' Quelle Steiner 1961 sowie Lechner 2017.

${ }^{86}$ Vgl. zu Beuys’ Konzeption des „Erweiterten Kunstbegriffs“ und seinem Konzept einer „Sozialen Plastik“ FIU und Borstel 1989. 
Bradley, W., und C. Esche, Hrsg. 2007. Art and social change. A critical reader. London: Tate Publishing und Afterall.

Cubitt, S. 2017. Finite media. Environmental implications of digital technologies. Durham und London: Duke University Press.

De Decker, K. 2009. The monster footprint of digital technology. Low-Tech Magazine 6. https://www.lowtechmagazine.com/2009/06/embodied-energy-of-digital-techno logy.html. Zugegriffen: 20. Juni 2020.

Deitcher, D. 2010. Social aesthetics. In Democracy: A project by Group Material, Hrsg. B. Wallis, 12-43. Seattle: Bay Press.

Demos, T. J. 2016. Decolonizing nature. Contemporary art and the politics of ecology. Berlin: Sternberg Press.

Dutli, R. 2012. Das Lied vom Honig. Eine Kulturgeschichte der Biene. Göttingen: Wallstein.

Eichel, H., Hrsg. 2015. 60 Jahre documenta. Die lokale Geschichte einer Globalisierung. Berlin: B \& S Siebenhaar.

Forschung Frankfurt. 2010. Nachhaltigkeit. Forschung Frankfurt. Wissenschaftsmagazin der Goethe-Universität Frankfurt a. M. 28:3. https://www.forschung-frankfurt.uni-frankf urt.de/36050840/Forschung_Frankfurt__Ausgaben_Archiv_2010. Zugegriffen: 20. Juni 2020.

Förster, Y. 2010. Alter Flugplatz Bonames/Former Bonames Airfield Frankfurt a. M., Deutschland/Germany. In Stadtgrün. Europäische Landschaftsarchitektur für das 21. Jahrhundert. Urban Green. European Landscape Design for the 21st Century. Hrsg. A. Becker und P. Cachola Schmal. Ausstellungskatalog Deutsches Architekturmuseum DAM Frankfurt a. M. 226-229. Basel: Birkhäuser.

Free International University (FIU) und S. von Borstel, Hrsg. 1989. Die Unsichtbare Skulptur. Zum erweiterten Kunstbegriff von Joseph Beuys. Stuttgart: Urachhaus.

Freistetter, F. 2015. Albert Einstein, das Sterben der Bienen und das ominöse Zitat. In ScienceBlogs. Astrodicticum Simplex 21.06.2015 https://scienceblogs.de/astrodicticumsimplex/2015/06/21/albert-einstein-das-sterben-der-bienen-und-das-ominoese-zitat/? all=1 Zugegriffen: 20. Juni 2020.

Gersmann, H., und B. Willms. 2010. „Die Welt kann man nicht Experten überlassen“. Interview mit Adrienne Goehler, Teil 1. In Zur Nachahmung empfohlen. Expeditionen in Ästhetik und Nachhaltigkeit. Hrsg. A. Goehler. Ausstellungskatalog Uferhallen Berlin und Lesebuch. 2 Bd. und Beilage. Band: Katalog. 5-11. Ostfildern-Ruit: Hatje Cantz.

Gillner, S. 2012. Stadtbäume im Klimawandel. Dendrochronologische und physiologische Untersuchungen zur Identifikation der Trockenstressempfindlichkeit häufig verwendeter Stadtbaumarten in Dresden. Diss. TU Dresden.

Goehler, A. Hrsg. 2010. Zur Nachahmung empfohlen. Expeditionen in Ästhetik und Nachhaltigkeit. Ausstellungskatalog Uferhallen Berlin und Lesebuch. 2 Bde. und Beilage. Ostfildern-Ruit: Hatje Cantz.

Goethe-Universität Frankfurt a. M., Hrsg. 2010. Frankfurter Bürger-Universität. Sommersemester 2010. Red. S. M. Hübner. Frankfurt a. M.: Goethe-Universität. https://www.bue rger.uni-frankfurt.de/50538486/BuergerUni-Broschuere-SS10.pdf. Zugegriffen: 20. Juni 2020.

Grober, U. 2010. Die Entdeckung der Nachhaltigkeit. Kulturgeschichte eines Begriffs. München: Kunstmann.

Groener, F., und R.-M. Kandler. Hrsg. 1987. Joseph Beuys. 7000 Eichen. Köln: König. 
Haraway, D. J. 2003. The companion species manifesto. Dogs, people, and significant otherness. Chicago: Prickly Paradigm Press.

Haraway, D. J. 2008. When species meet, 2008. Minneapolis: University of Minnesota Press.

Haraway, D. J. 2016. Staying with the trouble. Making kin in the chthulucene. Durham und London: Duke University Press.

Harlan, V., R. Rappmann, und P. Schata. 1980. Soziale Plastik. Materialien zu Joseph Beuys. Achberg: Achberger Verlagsanstalt (Erste Auflage: 1976).

Horn, H., und C. Lüllmann. 2006. Das große Honigbuch. Entstehung, Gewinnung, Gesundheit und Vermarktung. Dritte Auflage. Stuttgart: Kosmos-Franck.

Hülbusch, K. H., und N. Scholz. 1984. Joseph Beuys - 7000 Eichen zur documenta 7 in Kassel. „Stadtverwaldung statt Stadtverwaltung “. Ein Erlebnis- und gärtnerischer Erfahrungsbericht. Kassel: Kasseler Verlag.

Jung-Hoffmann, I., Hrsg. 1993. Bienenbäume, Figurenstöcke und Bannkörbe. Berlin: Fördererkreis der Naturwissenschaftlichen Museen Berlins.

Kagan, S. 2011. Art and sustainability. Connecting patterns for a culture of complexity. Bielefeld: transcript.

Kaufmann, C., Hrsg. 2015. Gerald Hintze. StadtMensch. Frankfurt a. M.: KANN-Verlag.

Kimpel, H. 1997. documenta. Mythos und Wirklichkeit. Köln: DuMont.

Kirksey, E., Hrsg. 2014. The multispecies salon. Durham und London: Duke University Press.

Kosut, L., und M. J. Moore. 2013. Buzz. Urban beekeeping and the power of the bee. New York: New York University Press.

Kosut, L., und M. J. Moore. 2014. Bees making art. Insect aesthetics and the ecological moment Humanimalia. A Journal of Human/Animal Interface Studies 5 (1): 1-25.

Kuni, V. 2006. Der Künstler als ,Magier “ und ,Alchemist“ im Spannungsfeld von Produktion und Rezeption. Aspekte der Auseinandersetzung mit okkulten Traditionen in der europäischen Kunst nach 1945. Phil. Diss. Marburg: Philipps-Universität. https://archiv.ub. uni-marburg.de/diss/z2006/0143/pdf/dvk.pdf sowie https://doi.org/10.11588/artdok.000 00192. Zugegriffen: 20. Juni 2020.

Kuni, V. 2010a. Nachhaltigkeit - (k)eine Kunst? Bäume pflanzen, Bienen züchten: ars longa als Gemeinschaftsprojekt. Forschung Frankfurt. Wissenschaftsmagazin der GoetheUniversität Frankfurt a. M. 28 (3): 4-9. https://www.forschung-frankfurt.uni-frankfurt.de/ 36050737/02Kuni.pdf. Zugegriffen: 20. Juni 2020.

Kuni, V. 2010b. „Resistance is Futile“. Von der Alien-Anthropologie zur CyborgEntomologie. In Kunst und Technik in medialen Räumen. Hrsg. S. Sanio. Saarbrücken: Pfau, 39-58.

Kuni, V. 2020. The plants are Sensing. In Spürtechniken. Von der Wahrnehmung der Natur zur Natur als Medium. = Medienobservationen - Sonderausgabe. Hrsg. B. Schneider und E. Zemanek. München: Ludwig-Maximilians-Universität, 30.04.2020 https:// www.medienobservationen.de/pdf/20200430Kuni6.pdf. Zugegriffen: 20. Juni 2020.

Kurt, H., und B. Wagner, Hrsg. 2002. Kultur - Kunst - Nachhaltigkeit. Die Bedeutung von Kultur für das Leitbild Nachhaltige Entwicklung. Bonn: Kulturpolitische Gesellschaft e. V. und Essen: Klartext.

Lavigne, E., K. Baudin, und J. Gregory, Hrsg. 2014. Pierre Huyghe. Ausstellungskatalog Centre Pompidou Paris u. a. München: Hirmer.

Lechner, G. 2017. Der soziale Organismus bei Rudolf Steiner und Rudolf Stolzmann. RoSE. Research on Steiner Education. 8 (1): 35-44. 
Loers, V., und P. Witzmann, Hrsg. 1993. Joseph Beuys. Documenta-Arbeit. Ausstellungskatalog Museum Fridericianum Kassel. Ostfildern: Hatje-Cantz.

Luttrell, J. 2017. Knowing the honeybee. A multispecies ethnography. MA-Thesis. Palmerston North: Massey University https://mro.massey.ac.nz/bitstream/handle/10179/12516/ 02_whole.pdf. Zugegriffen: 20. Juni 2020.

Miles, M. 2014. Eco-Aesthetics. Art, literature and architecture in a period of climate change. London: Bloomsbury.

Mosbrugger, V., G. Brasseur, M. Schaller, und B. Stribrny, Hrsg. 2012. Klimawandel und Biodiversität. Folgen für Deutschland. Darmstadt: WBG.

Ogunseitan, O. A., J. M. Schoenung, J. M. Saphores, und A. A. Shapiro. The electronics revolution. From e-wonderland to e-wasteland. Science 326: 5953 (30.10.2009), 670-671.

Olander, W. 1983. Social aesthetics. Art and social change, USA. AMAM Bulletin 40:2 (19821983). Oberlin: Allen Memorial Art Museum und Oberlin College, 61-69.

Pelletier, A., und P. E. Couton, Hrsg. 2019. Trees. Ausstellungskatalog Fondation Cartier pour l'Art Contemporain Paris. Paris: Fondation Cartier pour l'Art Contemporain und London: Thames \& Hudson.

Powers, R. 2018. The overstory. New York: W. W. Norton \& Company. Deutsch: Die Wurzeln des Lebens. Übs. G. Kempf-Allié und M. Allié. Frankfurt a. M.: Fischer.

Quaranta, D., und andere. 2009. Eva and Franco Mattes. 0100101110101101.ORG. Mailand: Charta.

Rödel, V. 2013. Baden unter Palmen. Flußbäder in Frankfurt a. M. 1800 - 1950. Frankfurt a. M.: Henrich Editionen.

Ropars, L., I. Dajoz, C. Fontaine, A. Muratet, und B. Geslin. 2019. Wild pollinator activity negatively related to honey bee colony densities in urban context. In PLOS ONE 12.09.2010 https://doi.org/10.1371/journal.pone.0222316. Zugegriffen: 20. Juni 2020.

Sauerländer, K., Hrsg. 2012. The book of books. documenta 13. = documenta 13. Band 1. Ostfildern: Hatje-Cantz.

Schlechtriemen, T. 2008. Metaphern als Modelle. Zur Organismus-Metaphorik in der Soziologie. In Visuelle Modelle. Hrsg. I. Reichle, S. Siegel und A. Spelten. München: Fink, $71-84$.

Schneider, B. 2018. Neue Formen der Klimakrisenwahrnehmung? Sprechende Bäume im Netz der Dritten Natur. Dritte Natur 1, 39-54 https://www.dritte-natur.de/magazin/det ails/neue-formen-der-klimakrisenwahrnehmung. Zugegriffen: 20. Juni 2020.

Schwarze, D. 2012. Meilensteine. Die documenta 1-13. Dritte erweiterte Auflage. Berlin: B \& S Siebenhaar.

Steiner, R. 1961. Die Kernpunkte der sozialen Frage in den Lebensnotwendigkeiten der Gegenwart und Zukunft. GA 23. Dornach: Rudolf-Steiner-Verlag.

Stiftung 7000 Eichen, Hrsg. 2012. 30 Jahre. Joseph Beuys. 7000 Eichen. Köln: König.

Strelow, H., Hrsg. 2004. Ökologische Ästhetik. Theorie und Praxis künstlerischer Umweltgestaltung. Basel: Birkhäuser.

Tautz, J. 2007. Phänomen Honigbiene. Heidelberg: Elsevier Spektrum Akademischer Verlag.

Ullrich, J. 2016. Tiere und Bildende Kunst. In Tiere. Kulturwissenschaftliches Handbuch. Hrsg. R. Borgards. Stuttgart: J. B. Metzler, 195-215.

Von Boenninghausen, C. M. F., Hrsg. 1863. Die Aphorismen des Hippokrates. Nebst den Glossen eines Homöopathen. Leipzig: Purfürst. 
Von Carlowitz, H. C. 1713. Sylvicultura oeconomica, oder haußwirthliche Nachricht und Naturmäßige Anweisung zur wilden Baum-Zucht. Leipzig: Braun.

Verena Kuni, Prof. Dr., Professur für Visuelle Kultur am Institut für Kunstpädagogik, Fachbereich Sprach- und Kulturwissenschaften der Goethe-Universität Frankfurt am Main. https://www.uni-frankfurt.de/66328036/Prof_Dr_Verena_Kuni kuni@kunst.uni-frankfurt.delverena@kuni.org

Open Access Dieses Kapitel wird unter der Creative Commons Namensnennung 4.0 International Lizenz (http://creativecommons.org/licenses/by/4.0/deed.de) veröffentlicht, welche die Nutzung, Vervielfältigung, Bearbeitung, Verbreitung und Wiedergabe in jeglichem Medium und Format erlaubt, sofern Sie den/die ursprünglichen Autor(en) und die Quelle ordnungsgemäß nennen, einen Link zur Creative Commons Lizenz beifügen und angeben, ob Änderungen vorgenommen wurden.

Die in diesem Kapitel enthaltenen Bilder und sonstiges Drittmaterial unterliegen ebenfalls der genannten Creative Commons Lizenz, sofern sich aus der Abbildungslegende nichts anderes ergibt. Sofern das betreffende Material nicht unter der genannten Creative Commons Lizenz steht und die betreffende Handlung nicht nach gesetzlichen Vorschriften erlaubt ist, ist für die oben aufgeführten Weiterverwendungen des Materials die Einwilligung des jeweiligen Rechteinhabers einzuholen.

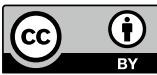

